Research Article

\title{
Comprehensive Analysis Identified ETV7 as a Potential Prognostic Biomarker in Bladder Cancer
}

\author{
Haimeng Li $(\mathbb{D}$, Yibo Zhang, and Shangyong Zheng $(\mathbb{D}$ \\ School of Medicine, Yunnan University, Kunming, China \\ Correspondence should be addressed to Shangyong Zheng; shangyong@ynu.edu.cn
}

Received 16 April 2021; Revised 12 October 2021; Accepted 27 October 2021; Published 9 December 2021

Academic Editor: Shufang Liang

Copyright ( 2021 Haimeng Li et al. This is an open access article distributed under the Creative Commons Attribution License, which permits unrestricted use, distribution, and reproduction in any medium, provided the original work is properly cited.

\begin{abstract}
Background. The tumor microenvironment (TME) plays a crucial role in the initiation and progression of cancer. Bladder cancer (BLCA) is a malignant tumor of the genitourinary system. Its heterogeneity results in significant differences in the prognosis of patients. To date, this is still a huge challenge for clinical treatment. In recent years, more and more evidence showed that dysregulation of transcription factors (TFs) plays an important role in tumor progression, invasion, and metastasis. Unfortunately, the role of TFs on the tumor microenvironment in bladder cancer is unclear. Methods. The original data of BLCA and corresponding adjacent tissues were obtained from The Cancer Genome Atlas (TCGA) database. TFs were downloaded from the Animal Transcription Factor DataBase (Animal TFDB). Intersection analysis was used to obtain TFs that were differentially expressed between tumor and adjacent tissues. Gene Set Cancer Analysis (GSCALite) and CIBERSORT software were used to reveal the key differentially expressed TFs (DE-TFs). Subsequently, UALCAN and Human Protein Atlas (HPA) databases were used to disclose the expression of key DE-TFs in BLCA. The $K-M$ curve divulged the relationship between the key DE-TFs and the patient's overall survival (OS), and the univariate and multivariate Cox regression analyses were conducted to explore independent prognostic factors. The cluster profiler package and Gene Set Enrichment Analysis (GSEA) were used for functional enrichment of genes related to the key DE-TFs. Finally, CIBERSORT software analyzed the immune landscape of BLCA. Results. We obtained a total of 117 BLCA-related DE-TFs. Among them, ETV7 was identified as the key DE-TFs due to its association with the autophagy activation pathway and various immune cells in cancer. Online databases of UALCAN and HPA indicated that ETV7 was overexpressed in tumors and negatively correlated with tumor severity. The $K-M$ curve showed that the OS of patients with high expression of ETV7 was poor, which indicated that it was an independent prognostic factor. Functional enrichment of 87 DEGs between ETV7-high and -low expression groups indicated that it was closely related to the immune response and the functions of a variety of immune cells. Finally, CIBERSORT results proved that the high and low expression of ETV7 also caused significant differences in the tumor immune microenvironment of patients. Conclusion. Overall, we proved that the transcription factor ETV7 was a novel prognostic factor, which may improve the individualized outcome prediction in BLCA by regulating the tumor immune microenvironment.
\end{abstract}

\section{Introduction}

Bladder cancer (BLCA) is a urinary tract malignancy with high morbidity and mortality, which is reported as the 10th most common cancer with an estimate 549,000 new cases and 200,000 death in 2018 [1]. Bladder cancer is a heterogeneous disease which consist of two subtypes nonmuscleinvasive bladder cancer (NMIBC) and muscle-invasive bladder cancer (MIBC). NMIBC, the majority of the BLCA do not penetrate the detrusor muscle layer which is not fatal but have high potential recurrence [2].
Despite the widespread use of multiple BLCA therapies, including surgery, radiation, chemotherapy, and Bacillus Calmette-Guerin (BCG), these therapies have not significantly improved 5-year survival over the past decade [2]. To date, the prognosis of BLCA patients largely depended on the histopathological diagnosis and tumor grading system, which raised the risk of BLCA recurrence. Therefore, it is very important to find some latent prognostic biomarkers for BLCA.

Transcription factors (TFs) bind to DNA and regulate transcription in a sequence-specific manner to form 
complex systems that direct genome expression. TFs are crucial for tumor progression, metastasis, and cancer metabolism through a wide range of physiological regulatory processes. Recent studies have shown that E2F1 is highly expressed in prostate cancer cells as an oncogene, and its expression level is closely related to the occurrence, development, and poor clinical prognosis of prostate cancer. EWS/FLI (TFS) binds GGAA-microsatellite sequences in regulation of the NROB1 gene as well as for Ewing sarcoma proliferation and anchorage-independent growth [3].

The E26 transformation-specific (ETS) family is one of the largest transcription factors involved in many biological processes, including cell differentiation, cell cycle control [4], cell migration [5], and cell proliferation [6]. This family consists of 28 human TFs that bind to similar DNA sequences of MAPK [7]. Multiple studies have shown that the abnormal expression in many members of the ETS family is closely associated with tumor initiation, progression, and metastasis in cancer [8-10].

In addition, the ETS family has been proved to be related to a wide range of immune-related biological process [11-13]. ETS-1, ETV5, PU1, GABP-1, and ETV6 have been shown to play critical roles in $\mathrm{T}$ cell differentiation [12]. ELF4 inhibits the proliferation of naive $\mathrm{CD}^{+} \mathrm{T}$ cells by enhancing the KLF4 expression [12, 14]. It is reported that ETV7 regulates the immune microenvironment in melanoma [15]. And it is an essential component of a rapamycininsensitive mTOR complex in cancers [16].

Tumor immune microenvironment (TIM) has been attracting interests over past years. The dysregulation of the immune microenvironment promotes the malignant progression of BLCA from NMIBC to MIBC. Interestingly, immunotherapy has played an important role in the treatment of BLCA, and Bacillus Calmette Guerin (BCG) remains to be the most efficacious intravesical medicament for NMIBC but with strong effects [17]. Therefore, it is very important to find a biomarker for bladder cancer tumor microenvironment.

In the current study, we systematically analyzed the expression profile and prognostic significance and role of ETV7 by integrating data from TCGA database, Animal Transcription Factor DataBase (AnimalTFDB), UALCAN, and Human Protein Atlas (HPA) databases, and our results indicated that ETV7 was a novel prognostic factor, which may improve the individualized outcome prediction in BLCA by regulating the tumor immune microenvironment.

\section{Materials and Methods}

2.1. Data Source. The database of The Cancer Genome Atlas (TCGA) was consulted to extract the RNA-seq data (FPKM format) and respective clinical data for a total of 408 BLCA patients and 19 adjacent samples. Clinical parameters, including gender, age, pathologic stage, pathologic $\mathrm{T}$ stage, pathologic $\mathrm{N}$ stage, and pathologic $\mathrm{M}$ stage, were also evaluated. 1665 human transcription factors were from the AnimalTFDB were posted on Supplementary Table S1 [18].
2.2. Differentially Expressed Gene (DEGs) Analysis. The $R$ package limma was utilized for gene expression data analysis [19]. Genes satisfying $\mid \log _{2}$ fold change (FC) $\mid$ of $>1$ and adjust (adj.) $P<0.05$ were perceived as DEGs in BLCA vs. adjacent tissue and high-ETV7 vs. low-ETV7. For the volcano plots and heatmaps, the ggplot 2 and pheatmap packages were employed to derive them, respectively.

DE-TFs were obtained through the intersection analysis of DEGs (BLCA vs. adjacent tissue) and TFs and then included in the univariate Cox regression analysis combined with the $K-M$ method. With $P<0.05$ as the threshold to obtain candidate DE-TFs.

2.3. GSCALite. GSCALite (http://bioinfo.life.hust.edu.cn/ web/GSCALite/) was applied in the present study to provide a web-based analysis candidate DE-TFs and cancer pathways. This includes differential expression and survival analysis, the correlation between gene expression and cancer pathways, and other analyses [20]. All the candidate DE-TFs were uploaded to the GSCALite website with the aim of analyzing the correlation between cancer pathways and candidate DE-TFs using the TCGA-BLCA samples $(N=414)$.

2.4. The Human Protein Atlas (HPA). The HPA is mapping all the human proteins in 64 cell lines, 48 human normal tissues, and 20 tumor types [21]. The key DE-TF, ETV7, was submitted to the HPA database, and the protein expression in BLCA was explored. The tissues information used in this study were as followed: patient ID: 1761 , male, age 51, urinary bladder (T-74000), normal tissue, NOS (M-00100); patient ID: 2053, male, age 51, urinary bladder (T-74000), lymph node (T-08000), urothelial carcinoma, and high grade (M-812033).

2.5. UALCAN. UALCAN is a web resource designed to analyze cancer transcriptome data in an interactive way [22]. In this study, ETV7 was presented to UALCAN, and the expression of its mRNA in TCGA-BLCA samples was investigated. Moreover, any correlation of the ETV7 expression with the clinicopathological features was also estimated. $P$ $<0.05$ suggested a meaningful variation.

2.6. Independent Validation of ETV7 Expression Levels in Multiple Databases. To enhance the reliability of the results of this study, we opted for three different databases, namely, the Gene Expression Omnibus (GEO, http://www.ncbi.nlm .nih.gov/geo/) database, the online Metabolic gEne RApid Visualizer (MERAV) database (http://merav.wi.mit.edu/), and the tumor immune estimation resource (TIMER) online database (http://timer.cistrome.org/) to further validate the differences in ETV7 expression between BLCA and normal tissues. For the GEO database, we downloaded the GSE7476 dataset (https://www.ncbi.nlm.nih.gov/geo/query/ acc.cgi?acc=GSE7476) containing 3 normal bladder samples and 9 BLCA samples. Based on RNA sequencing data, the difference in the ETV7 expression between normal tissues and BLCA was detected by the Wilcoxon test method. The publicly available website, MERAV, was designed to analyze the human gene expression in 4454 arrays and normalize all 


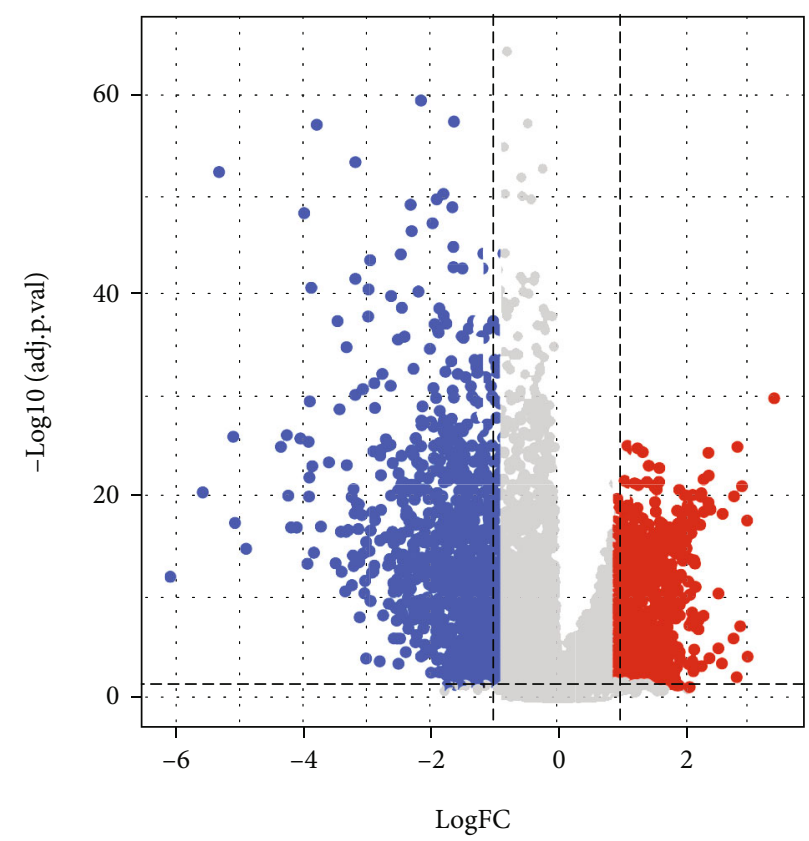

Significant

- Down

- Not

- Up

(a)

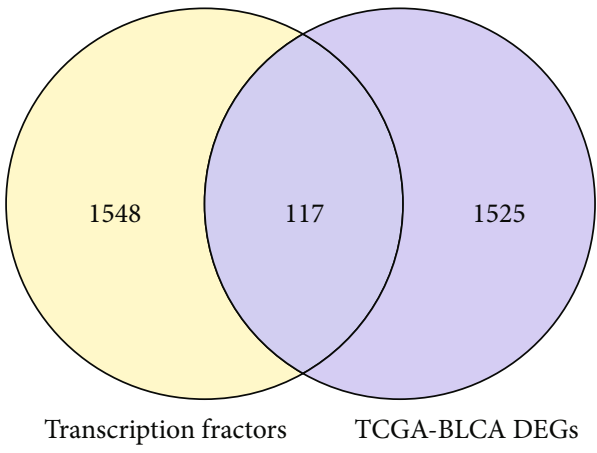

(b)

FIgUre 1: Continued. 


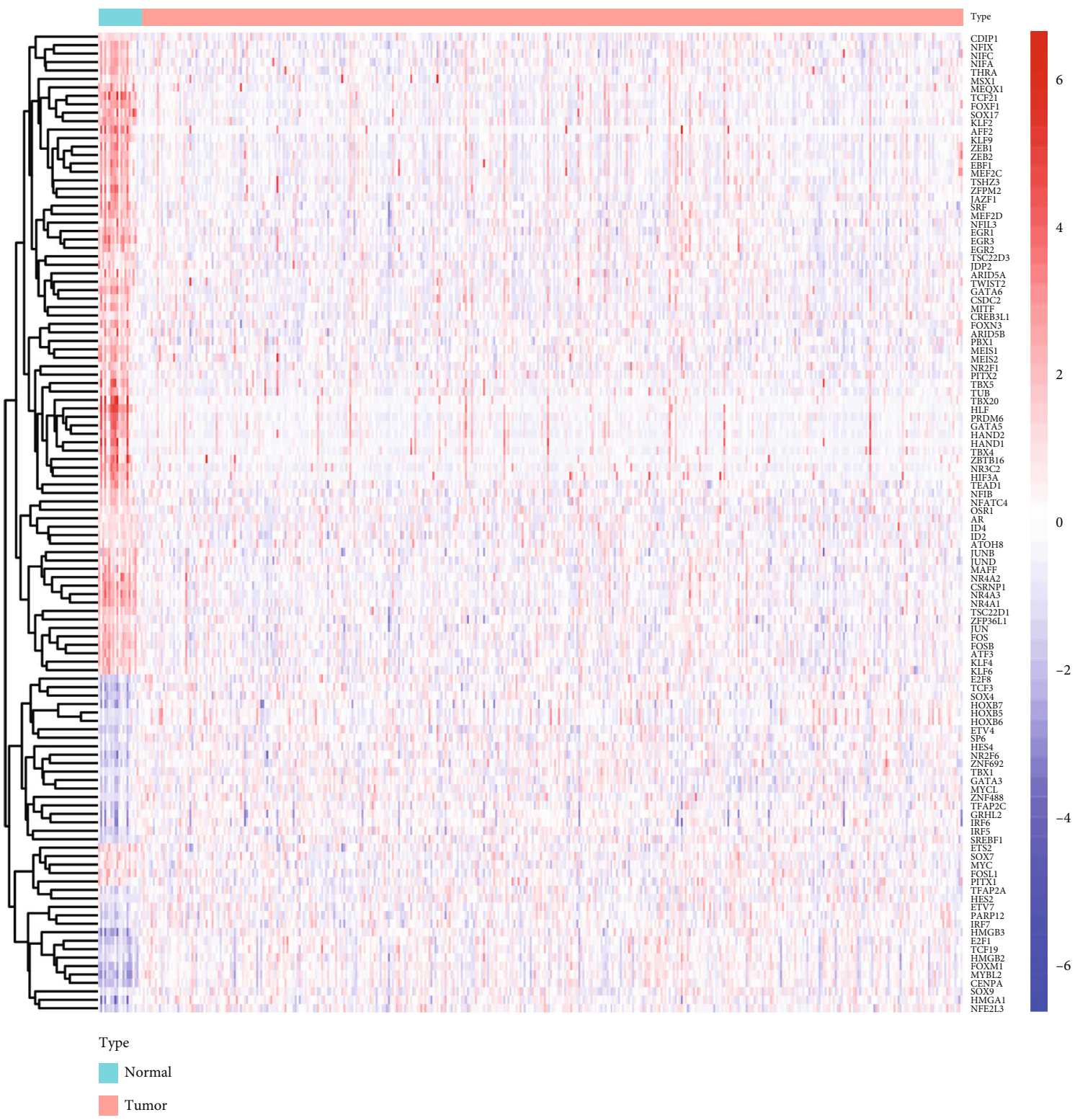

(c)

FIGURE 1: Distribution and intersection of DE-TFs in bladder cancer. (a) Distribution of the DEGs in the TGCA-BLCA database. (b) Venn plots showing 117 common DE-TFs shared by TFs and TCGA-BLCA DEGs. (c) Heatmap shows the expression levels of 117 DE-TFs, the red indicated tumor sample, and the blue indicated normal sample.

arrays together to generate a database $[23,24]$ for the ETV7 expression differential validation. TIMER is a comprehensive resource for systematic analysis of immune infiltration of different cancer types [25]. Not as well known, the database also has cancer exploration capabilities, which can detect gene expression differences between tumor and normal groups.

2.7. Enrichment Analysis. Gene Ontology (GO) and the Kyoto Encyclopedia of Genes and Genomes (KEGG) were administered in clusterProfiler to enable enrichment analysis for all DEGs between the high-ETV7 and low-ETV7 groups. Furthermore, all the genes in the high-ETV7 and low-ETV7 groups were submitted to the GSEA and chose the C5 (c5.go.v7.2.symbols.gmt) and C2 (c2.cp.kegg.v7.2.symbols.gmt) subcollection downloaded from the Molecular Signatures Database (http://software.broadinstitute.org/ gsea/msigdb/index.jsp) as the reference gene sets.

2.8. Assessment of Tumor-Infiltrating Immune Cells. With the CIBERSORT algorithm, the fractions of infiltrating immune cells in each BLCA sample were derived for the high-ETV7 and low-ETV7 groups. CIBERSORT is designed to calculate the cellular composition of complex tissues working from the standardized gene expression data [26]. This method energizes the abundance of specific cell types [27]. The results generated by CIBERSORT were regarded as accurate when $P<0.05$ was met [28]. 


$\begin{array}{lrl} & \text { Pvalue } & \text { Hazard Ratio } \\ \text { NFIA } & 0.031 & 1.248(1.02-1.525) \\ \text { EGR2 } & 0.012 & 1.216(1.044-1.416) \\ \text { CSDC2 } & 0.026 & 1.188(1.02-1.383) \\ \text { MYC } & 0.034 & 1.129(1.009-1.262) \\ \text { ID2 } & 0.006 & 0.836(0.736-0.949) \\ \text { ETV7 } & <0.001 & 0.774(0.672-0.892) \\ \text { PITX1 } & 0.042 & 1.115(1.004-1.238) \\ \text { HOXB5 } & <0.001 & 0.777(0.672-0.897) \\ \text { HES4 } & 0.010 & 0.858(0.763-0.964) \\ \text { HOXB6 } & 0.006 & 0.820(0.712-0.946) \\ \text { GATA3 } & 0.009 & 0.907(0.842-0.976) \\ \text { TBX1 } & <0.001 & 0.851(0.773-0.936) \\ \text { SP6 } & 0.004 & 0.852(0.763-0.951)\end{array}$

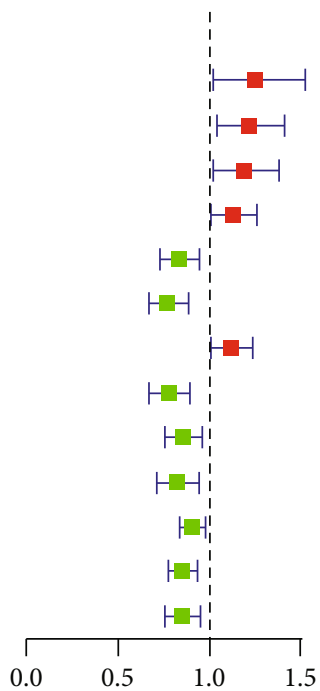

Hazard Ratio

(a)

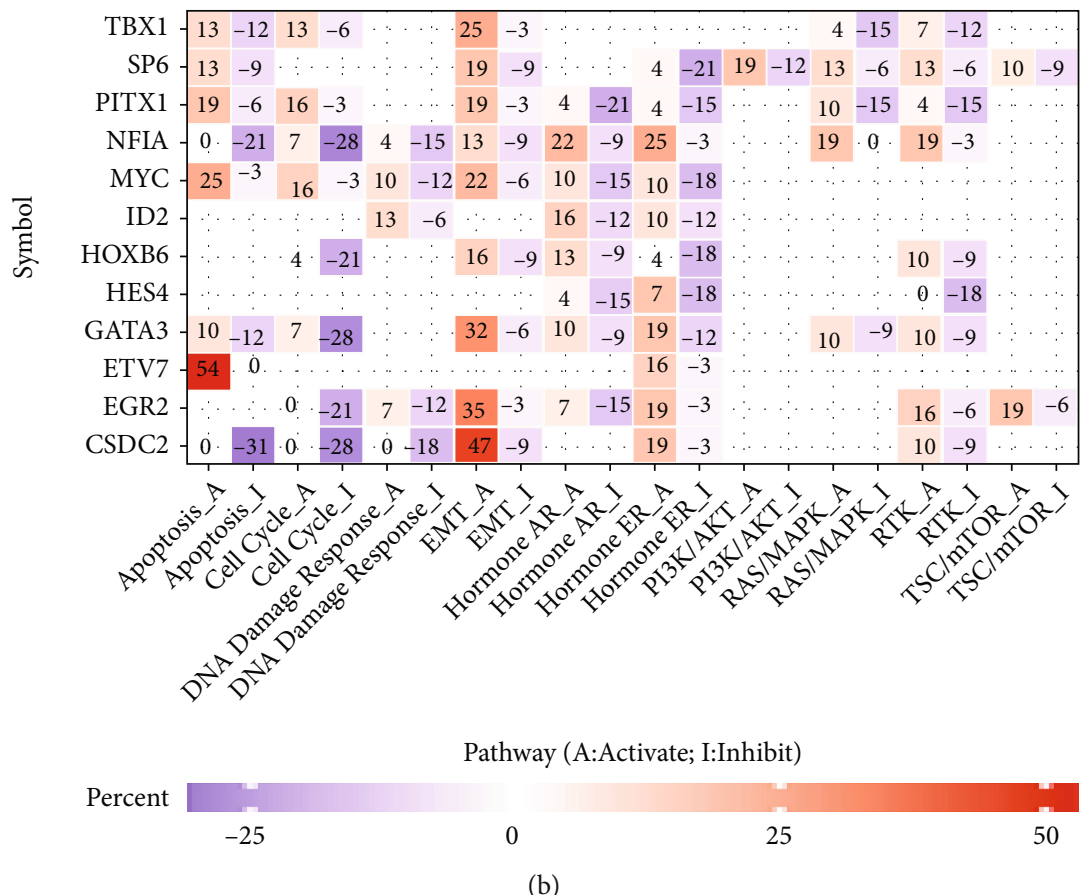

(b)

Figure 2: Continued. 


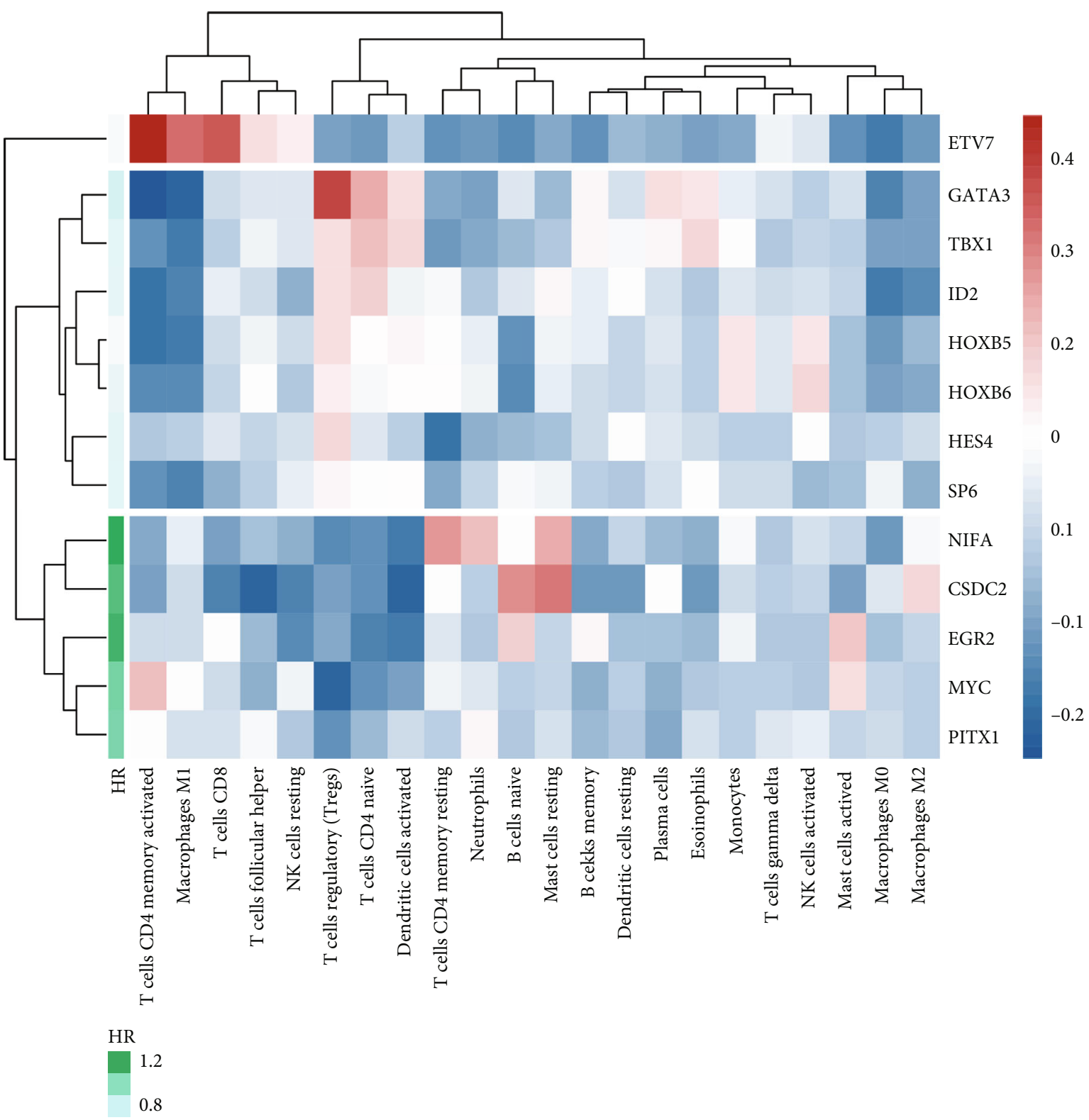

(c)

FIgURE 2: Screening of 13 TFs associated with immune infiltrating. (a) Univariate Cox analysis with 117 DE-TFs listing the top significant factors with $P<0.005$. (b) The correlation of 13 candidate DE-TFs with various cancer pathway. The higher the absolute value, the stronger the correlation with this cancer pathway. (c) The correlation between the expression of $13 \mathrm{DE}-\mathrm{TFs}$ and immune infiltrating cell. The color represents the Pearson correlation, and the value represents the strength of the correlation.

2.9. Statistical Analysis. In this study, statistical analyses were carried out using the $R$ package. The correlation between 13 candidate DE-TFs and immune cells was revealed by Pearson correlation analysis. The log-rank test was invoked in the $K-M$ survival analysis. Independent factors were established by univariate and multivariate Cox regression analysis. All $P<0.05$ made a significant difference statistically.

\section{Results}

3.1. Screening of DE-TFs in the TCGA-BLCA Database. The analysis of the TCGA-BLCA database and comparison of the BLCA group with a normal group identified 1663 (759 upregulated and 904 downregulated) DEGs. The distribution of the DEGs in the TCGA-BLCA database was shown in Figure 1(a). The upregulated and downregulated DEGs of the TCGA-BLCA database were shown in Supplementary Tables S2 and S3, respectively. A total of 117 DE-TFs were obtained by further analysis of the DEGs and 1665 TFs (Figure 1(b)). All of the DE-TFs were displayed in Supplementary Table S4. Also, we showed the data by a heat map (Figure 1(c)).

3.2. The Key DE-TF Screening of BLCA. We explored the relationship between the DE-TFs and OS by utilizing univariate Cox analysis combined with the $K-M$ curve. Of note, 


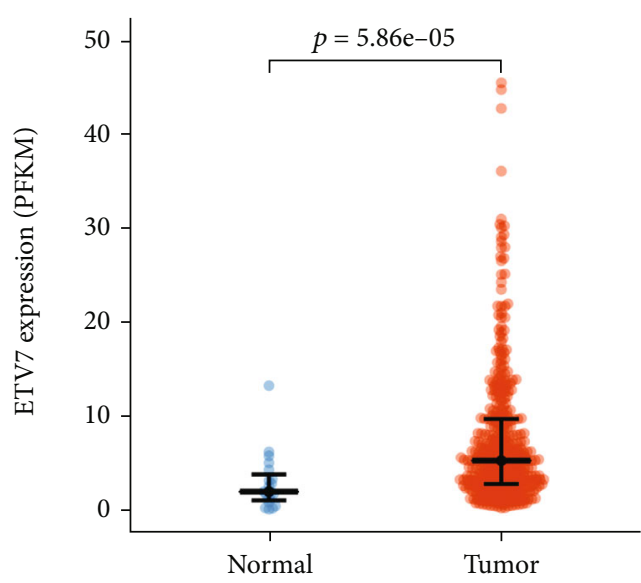

(a)

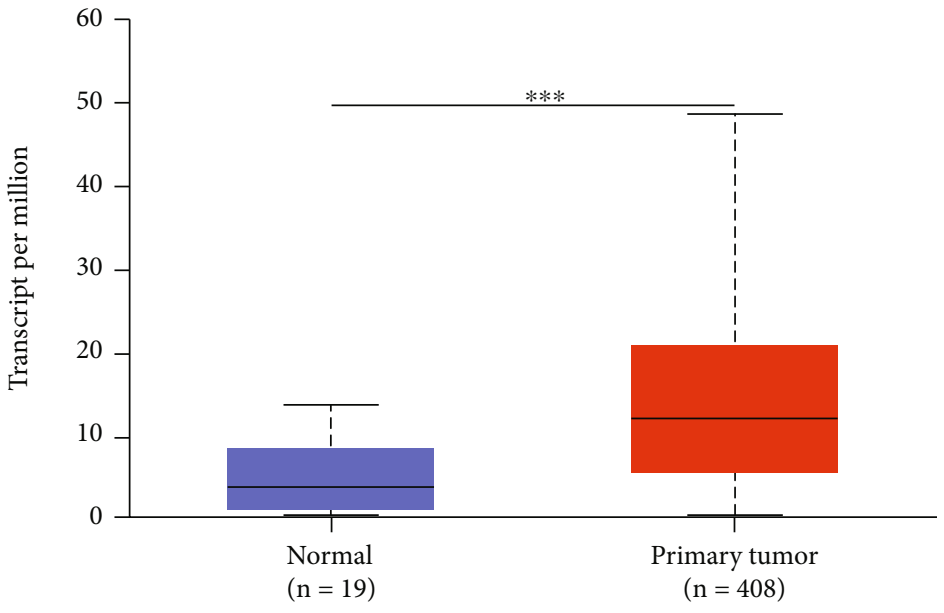

TCGA Samples

(b)
Normal ID: 1761

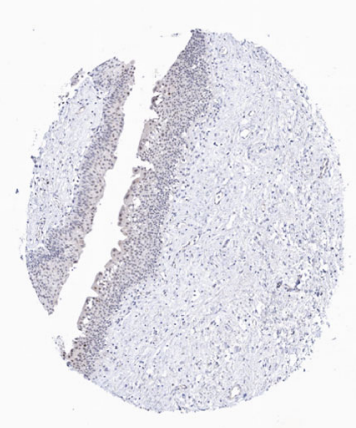

Staining: Not detect

Antibody: HPA029033

(c)

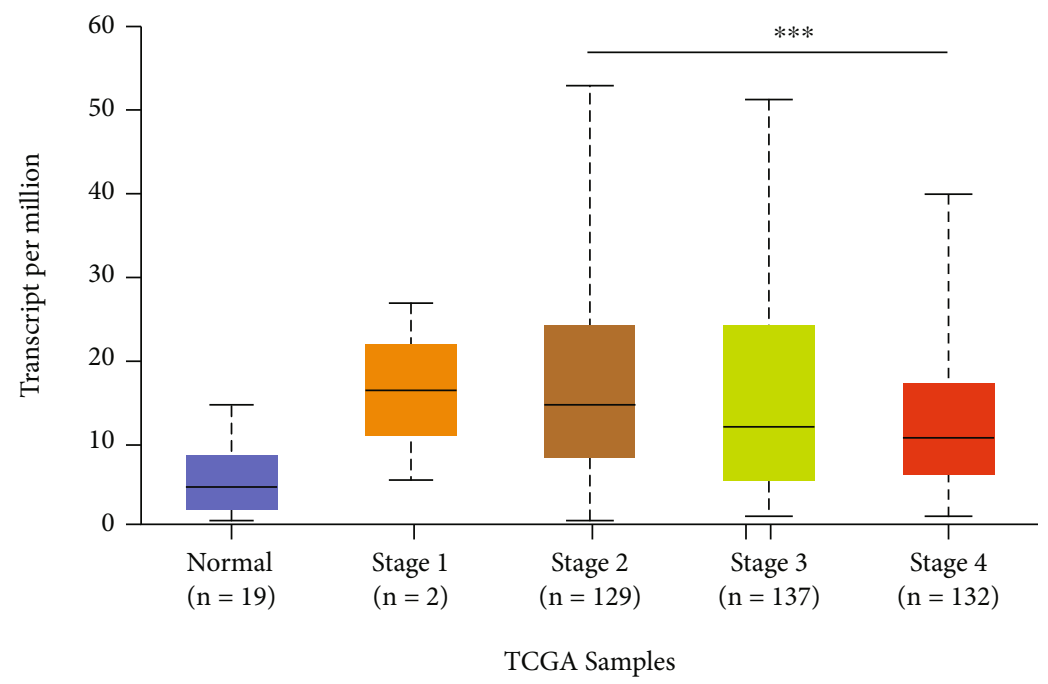

(d)

Figure 3: Continued. 


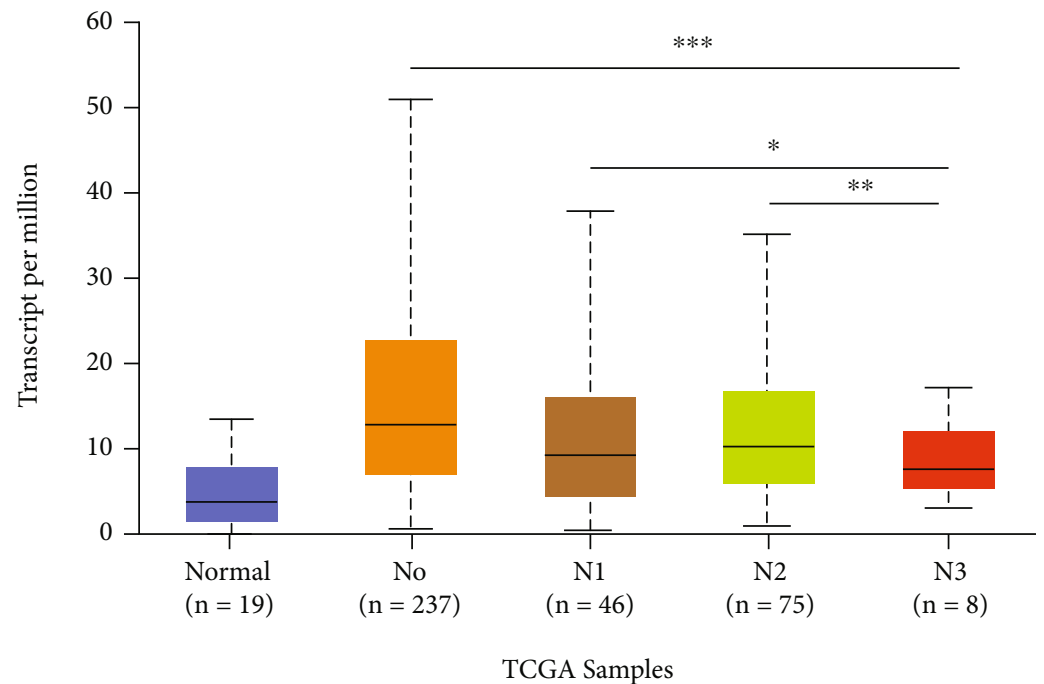

(e)

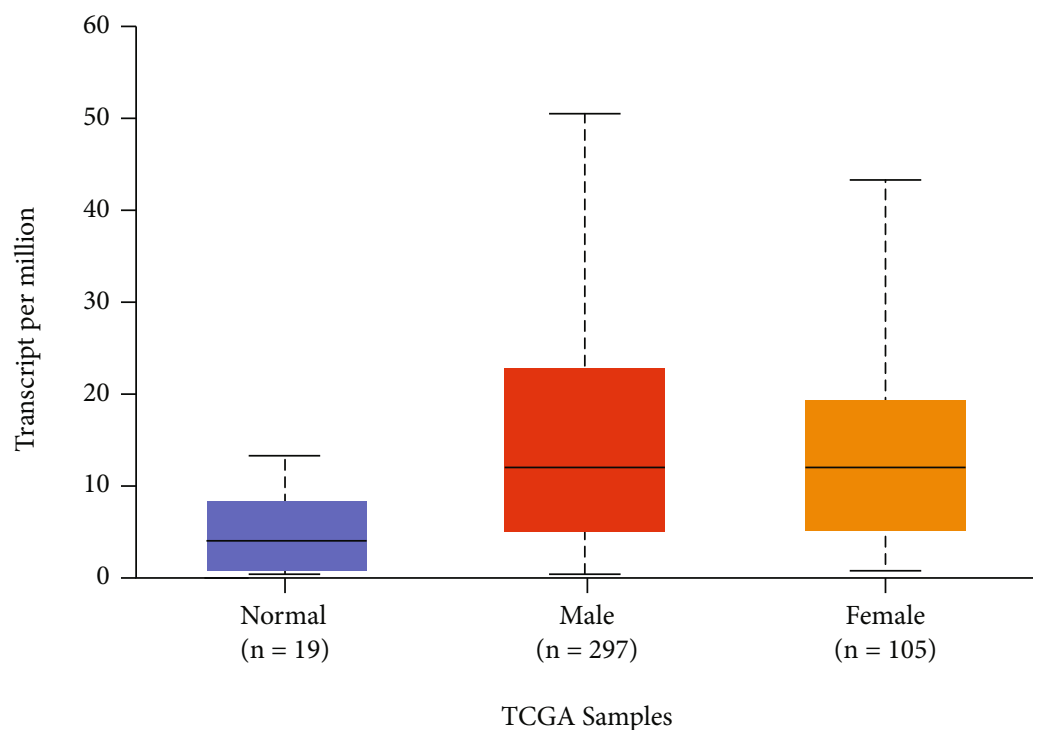

(f)

Figure 3: Continued. 


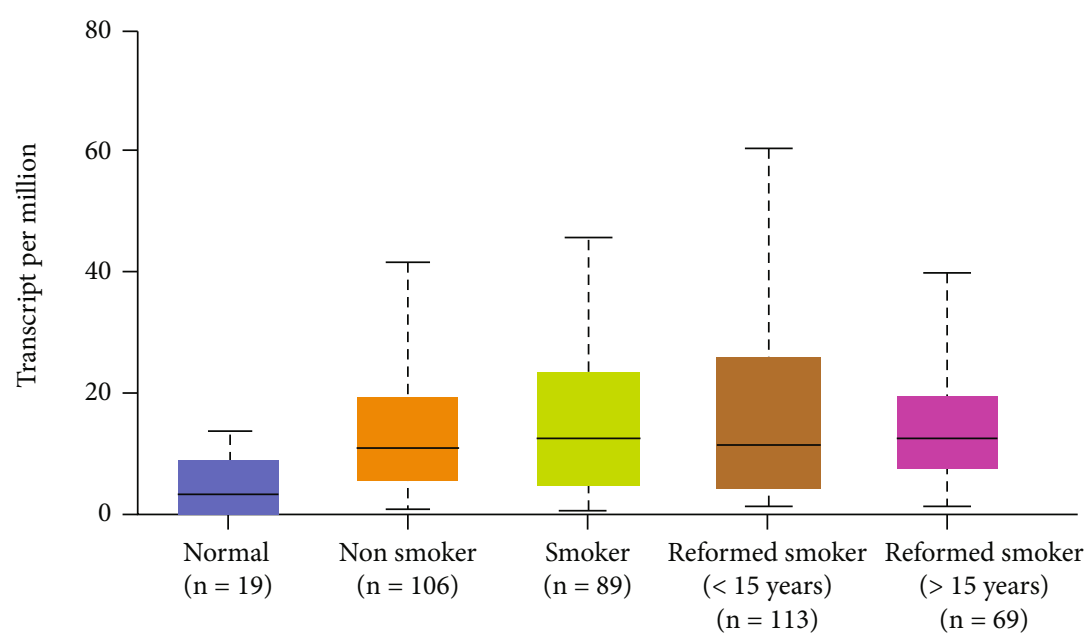

TCGA Samples

(g)

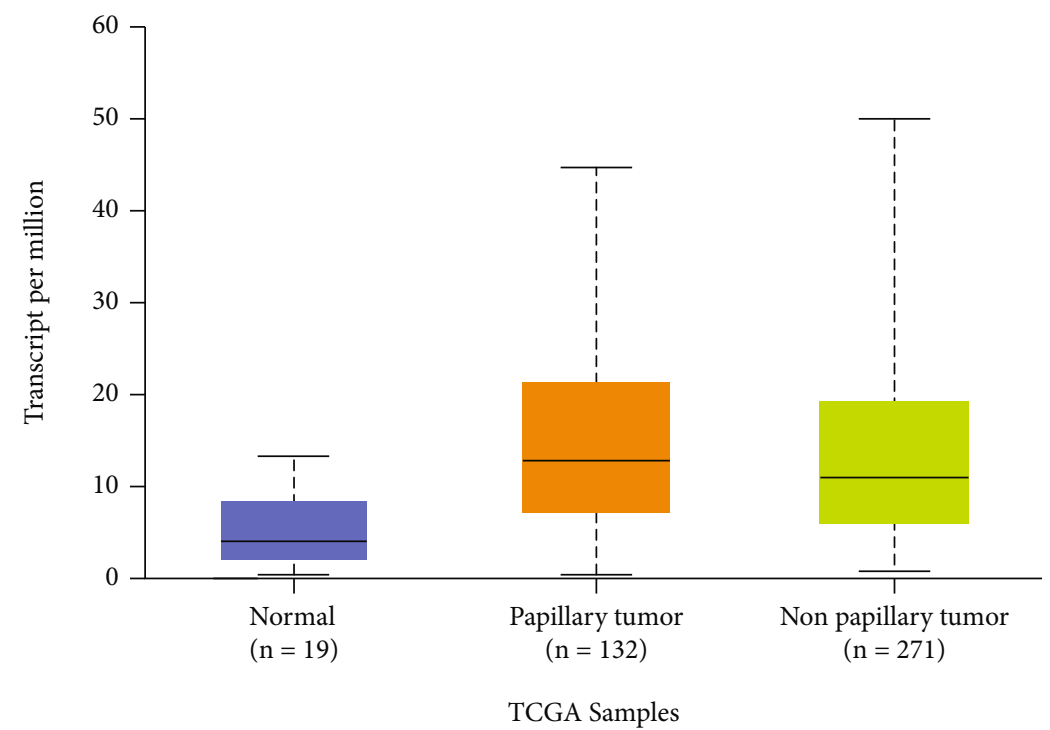

(h)

Figure 3: ETV7 differential expression in BLCA tissues. (a) ETV7 expression in tumor and normal samples. (b) The mRNA expression pattern of ETV7 measured by UALCAN database. (c) Immunohistochemical levels of ETV7 in BLCA samples. (d)-(h) Correlation between expression of ETV7 and clinicopathological staging characteristics.

among the 117 DE-TFs, 13 met the criteria for statistical significance via the $P<0.05$ (Supplementary Table S5). NFIA, EGR2, CSDC2, MYC, and PITX were considered risk factors $(\mathrm{HR}>1)$, and the remaining 7 DE-TFs were protective factors $(\mathrm{HR}<1)$ (Figure $2(\mathrm{a}))$.

To learn more about the potential worked of the 13 candidate DE-TFs in BLCA, we first analyzed the relationship between DE-TFs and the activities of various cancer pathways using the GSEALite website. It was apparent that ETV7 played a more prominent role in apoptosis activation. Also, we found that CSDC2 was related to activation of the EMT pathway and related to inhibition of the apoptosis and cell cycle pathways (Figure 2(b)). Positive numbers represented activation and negative numbers represented inhi- bition. The higher the absolute value, the stronger the correlation with this cancer pathway. A correlation between the expression of 13 DE-TFs and immune infiltrating cells was also evaluated, which showed that the most DE-TFs had a strong correlation with immune infiltrating cells. Strikingly, the cluster analysis showed that ETV7 was unique and had the strongest positive correlation with T cells CD4 memory activated. Coincidentally, there are also inextricable links between autophagy and T cells CD4 memory activated [29-31] (Figure 2(c)). The color represents the Pearson correlation, and the value represents the strength of the correlation. In summary, ETV7 may play a vital role in the occurrence and development of BLCA. We regarded it as a key DE-TF and carried out further research. 


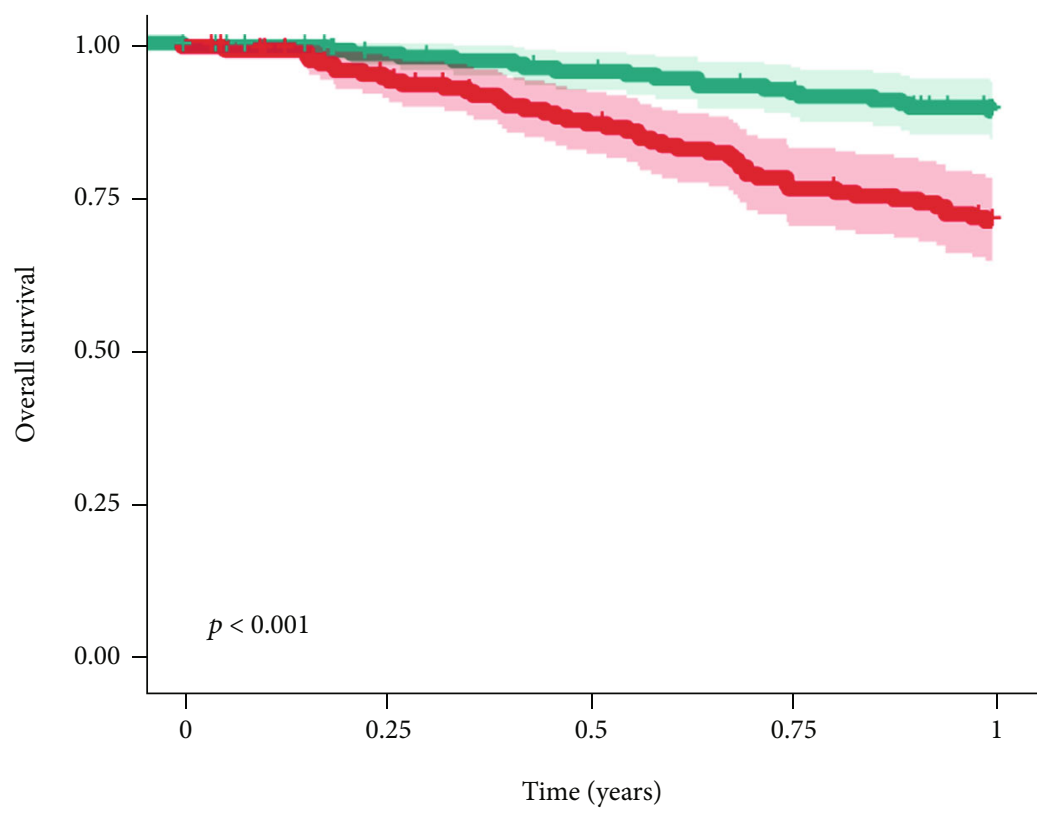

Low -\begin{tabular}{ccccc} 
Number at risk & & & \\
184 & 172 & 165 & 158 & 144 \\
185 & 167 & 150 & 131 & 119 \\
\cline { 1 - 4 } & 0.25 & 0.5 & 0.75 & 1 \\
& & & &
\end{tabular}

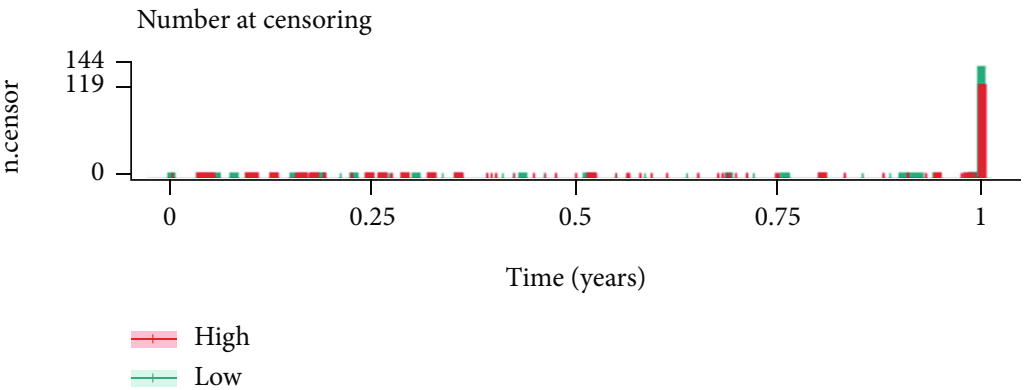

(a)

Figure 4: Continued. 


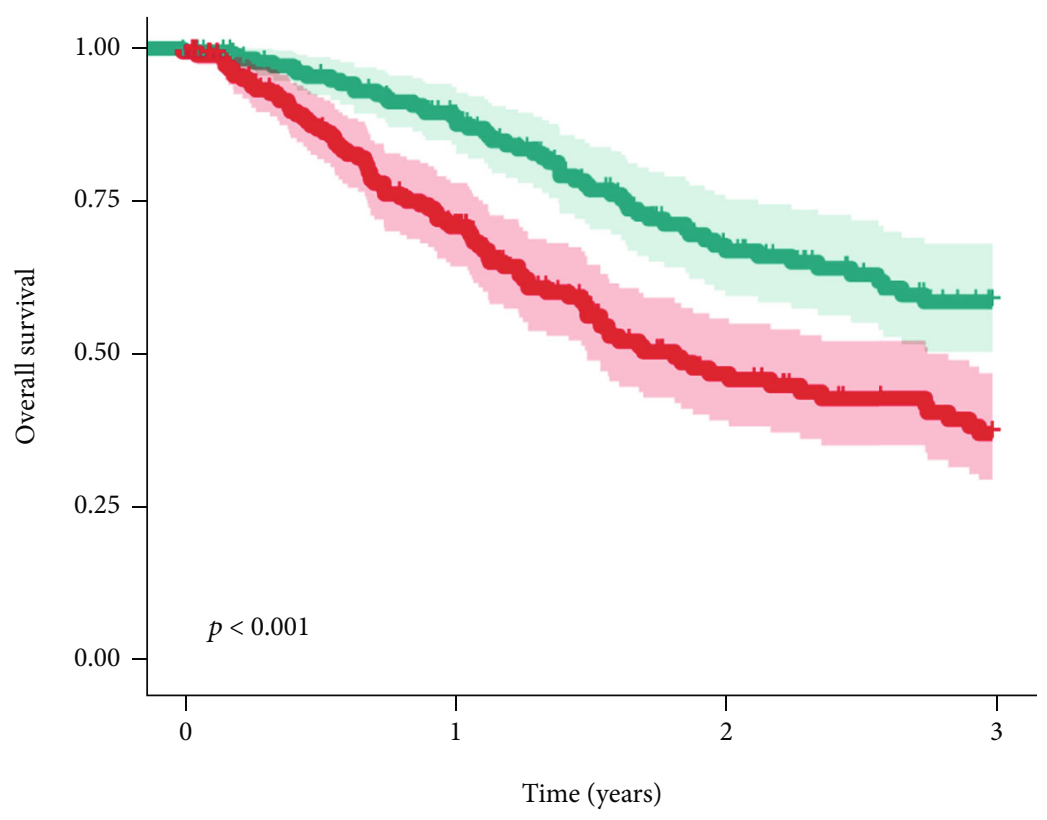

\begin{tabular}{|c|c|c|c|c|}
\hline \multicolumn{5}{|c|}{ Number at risk } \\
\hline Low - & 184 & 144 & 79 & 47 \\
\hline High - & 185 & 119 & 51 & 33 \\
\hline & $T$ & $T$ & $T$ & $T$ \\
\hline & 0 & 1 & 2 & 3 \\
\hline
\end{tabular}

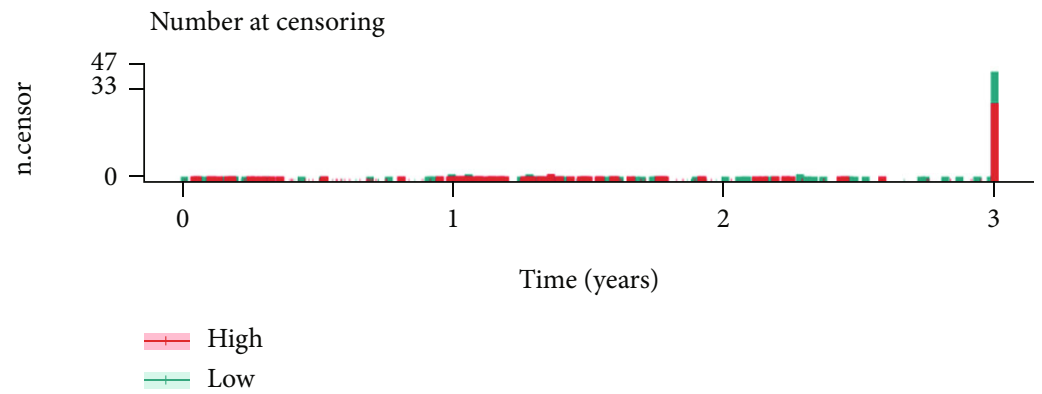

(b)

Figure 4: Continued. 


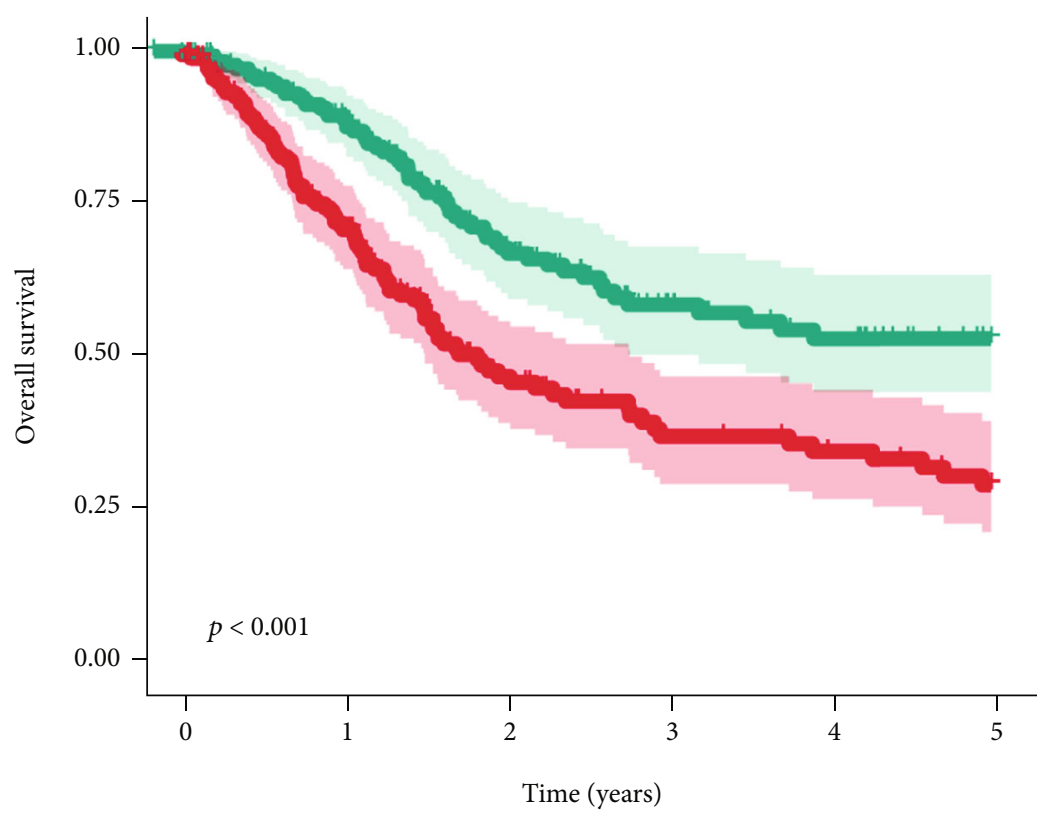

Low -\begin{tabular}{rrrrrr} 
Number at risk \\
184 & 144 & 79 & 47 & 37 & 24 \\
185 & 119 & 51 & 33 & 27 & 21 \\
\hline 1 & 1 & 2 & 3 & 4 & 5
\end{tabular}

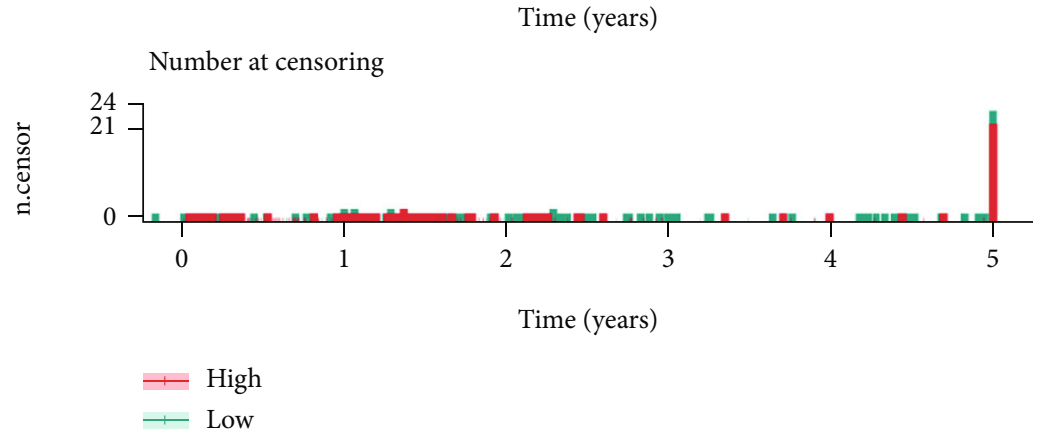

(c)

Figure 4: Continued. 


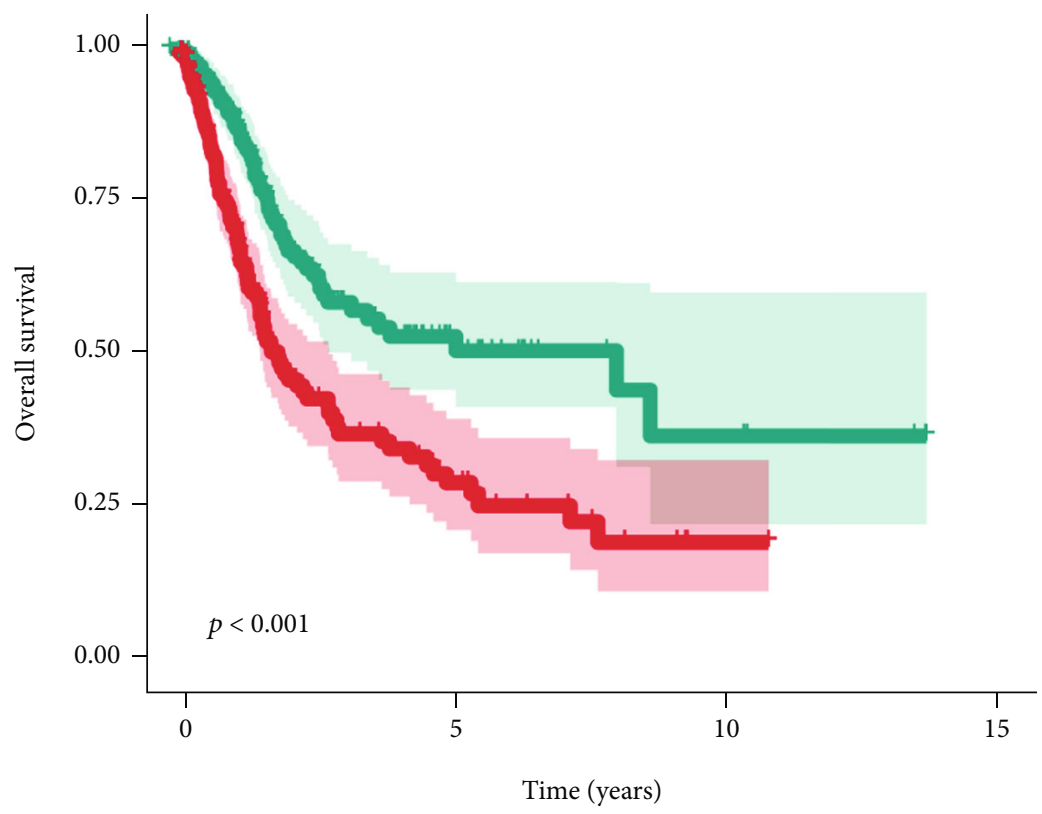

Number at risk

Low -\begin{tabular}{cccc}
184 & 24 & 5 & 0 \\
185 & 21 & 1 & 0 \\
\hline 0 & 5 & 10 & 1 \\
& & & 1
\end{tabular}

Number at censoring

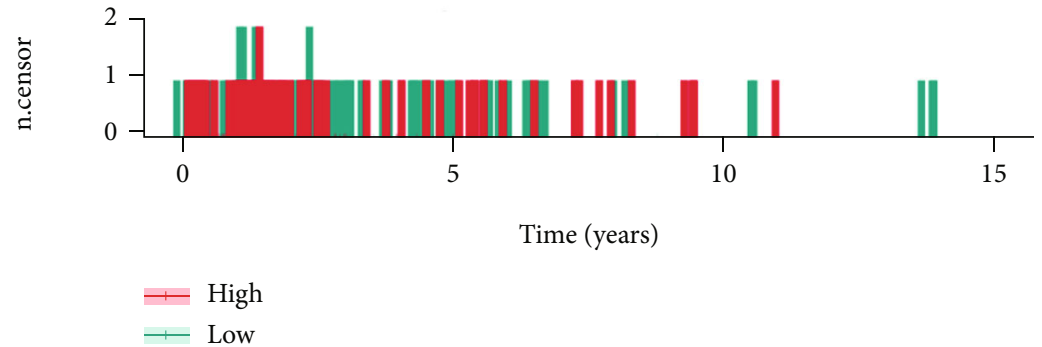

(d)

$\begin{array}{lrr} & \text { Pvalue } & \text { Hazard ratio } \\ \text { Age } & <0.001 & 1.034(1.017-1.051) \\ \text { Gender } & 0.299 & 0.837(0.597-1.172) \\ \text { Pathologic_stage } & <0.001 & 1.787(1.452-2.198) \\ \text { Pathologic_t } & <0.001 & 1.716(1.369-2.152) \\ \text { pathologic_m } & 0.031 & 1.186(1.016-1.386) \\ \text { Pathologic_n } & <0.001 & 1.219(1.093-1.359) \\ \text { ETV7 } & 0.004 & 0.963(0.939-0.988)\end{array}$

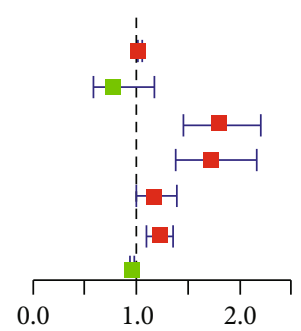

Hazard ratio

(e)

Figure 4: Continued. 


$\begin{array}{lrr} & \text { Pvalue } & \text { Hazard ratio } \\ \text { Age } & <0.001 & 1.032(1.015-1.049) \\ \text { Gender } & 0.132 & 0.768(0.545-1.083) \\ \text { Pathologic_stage } & 0.094 & 1.259(0.962-1.649) \\ \text { Pathologic_t } & 0.007 & 1.479(1.115-1.963) \\ \text { Pathologic_m } & 0.034 & 1.189(1.013-1.395) \\ \text { Pathologic_n } & 0.007 & 1.206(1.052-1.383) \\ \text { ETV7 } & 0.019 & 0.971(0.948-0.995)\end{array}$

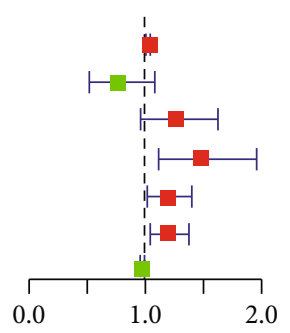

Hazard ratio

(f)

Figure 4: Prognostic analysis of ETV7 in BLCA patients. (a)-(d) The association between ETV7 and the prognosis of BLCA patients. (e, f) Univariate and multivariate Cox analysis of clinical characteristics and the ETV7 gene.

3.3. Profiles of ETV7 Expression in BLCA. BLCA tissues exhibited a significantly higher level of the ETV7 $(P=5.86 E-05)$ expression (Figure 3(a)) than normal tissues. Naturally, the expression pattern of ETV7 mRNA was characterized by the UALCAN database. As was shown in Figure 3(b), the mRNA expression of the key DE-TF was found to be significantly upregulated in primary BLCA tissues compared to normal samples (all $P<0.05$ ). Furthermore, analysis of the dysregulated ETV7, using HPA, displayed increased protein levels in BLCA, with respect to specimens from normal controls (Figure 3(c)). Aggregately, a robust affirmation of the importance of ETV7 in BLCA was noted in these findings.

Following the discovery of mRNA and protein overexpression in BLCA patients, we next analyzed the relationship between the mRNA expression of ETV7 with clinicopathological parameters of BLCA patients by UALCAN, including a patient's gender, smoking habit, tumor type, tumor stage, and $\mathrm{N}$ stage. As was shown in Figure 3(d), the mRNA expression of ETV7 was remarkably correlated with patients' cancer stages, where patients in advanced stages of cancer tended to express lower levels of ETV7. The highest expression of ETV7 was found in stage 1. Similarly, as was illustrated in Figure 3(e), the ETV7 expression levels were significantly related to $\mathrm{N}$ stages, and, as the $\mathrm{N}$ stage increased, the expression of ETV 7 tended to be lower. The highest mRNA expression of ETV7 was noted in the N0 stage. The reason why the mRNA expression of ETV7 in the N1 stage seemed to be lower than that in the N2 stage may be due to the small sample size (only 46 BLCA patients were at the N1 stage). However, the expression level of EVT7 had no significant correlation with the patient's gender, smoking habits, and tumor type (Figures 3(f)-3(h)). These results suggested that EVT7 was significantly associated with clinicopathological parameters in BLCA patients.

To ensure the accuracy of the results, we further examined the expression differences of ETV7 in BLCA and normal groups from the GSE7476 dataset, MERAV online database, and TIMER database, respectively. Consistent with the results in TCGA database, as shown in Supplementary Figure 1, all three independent external databases indicated that ETV7 was significantly overexpressed in BLCA $(\mathrm{P}<0.05)$.
3.4. Prognostic Value of ETV7 in BLCA. The association between $E T V 7$ and the prognosis of BLCA patients was subsequently reviewed. The $1-, 3-$, and 5-year $K-M$ curves showed that there was a significant difference in survival between the high and low expression groups of ETV7 (Figures 4(a)-4(c); all $P<0.001)$. OS analysis revealed that BLCA patients with a high level of $\operatorname{ETV} 7(P<0.001)$ had a poor OS rate (Figure 4(d)). Thus, ETV7 was a potential prognostic biomarker for BLCA.

The interaction among clinicopathological variables, ETV7, and OS was primarily adjusted by a univariate Cox regression model, following which, and salient variables $(P<0.05)$ were employed for multivariate Cox regression model estimation. In the univariate Cox analysis, we limited this to include gender, age, pathologic stage, pathologic $\mathrm{T}$ stage, pathologic $\mathrm{N}$ stage, pathologic $\mathrm{M}$ stage, and ETV7. Age $(P<0.001)$, pathologic stage $(P<0.001)$, pathologic $\mathrm{T}$ stage $(P<0.001)$, pathologic $\mathrm{N}$ stage $(P<0.001)$, pathologic $M$ stage $(P=0.031)$, and ETV7 $(P=0.004)$ were significant but gender was not $(P=0.299)$ (Figure 4(e); Supplementary Table S6). In the multivariate analysis, age $(P<0.001)$, pathologic $\mathrm{T}$ stage $(P=0.007)$, pathologic $\mathrm{N}$ stage $(P=0.007)$, pathologic $M$ stage $(P=0.034)$, and ETV7 $(P=0.019)$ were all remained significant (Figure $4(\mathrm{f})$; Supplementary Table S7).

3.5. Functional Enrichment of DEGs between ETV7-High and -Low Expression Groups. DEGs between high- and lowETV7 expression groups were screened by adj. $P<0.05$ and $\left|\log _{2} \mathrm{FC}\right|>1$. A total of 87 DEGs (82 upregulated and 5 downregulated) were identified in the high ETV7 group (Figure 5(a)) and visualized using a heat map (Figure 5(b)). The detailed DEGs were illustrated in Supplementary Table S8.

GO and KEGG analysis was carried out using the clusterProfiler package to investigate the potential capabilities of the DEGs identified above. Significance $(P<0.05)$ was achieved for a total of 227 GO terms of biological process (BP), 51 GO terms of cellular component (CC), and 25 GO terms of molecular function (MF). Top 10 GO BP terms indicated that the DEGs were primarily enriched in "response to interferon-gamma," "cellular response to interferon-gamma," and "interferon-gamma-mediated signaling 


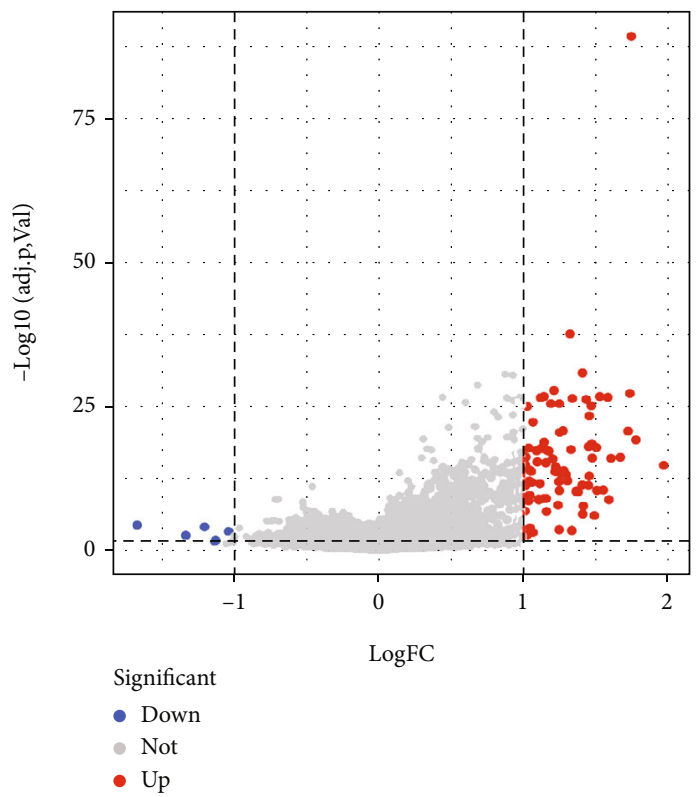

(a)

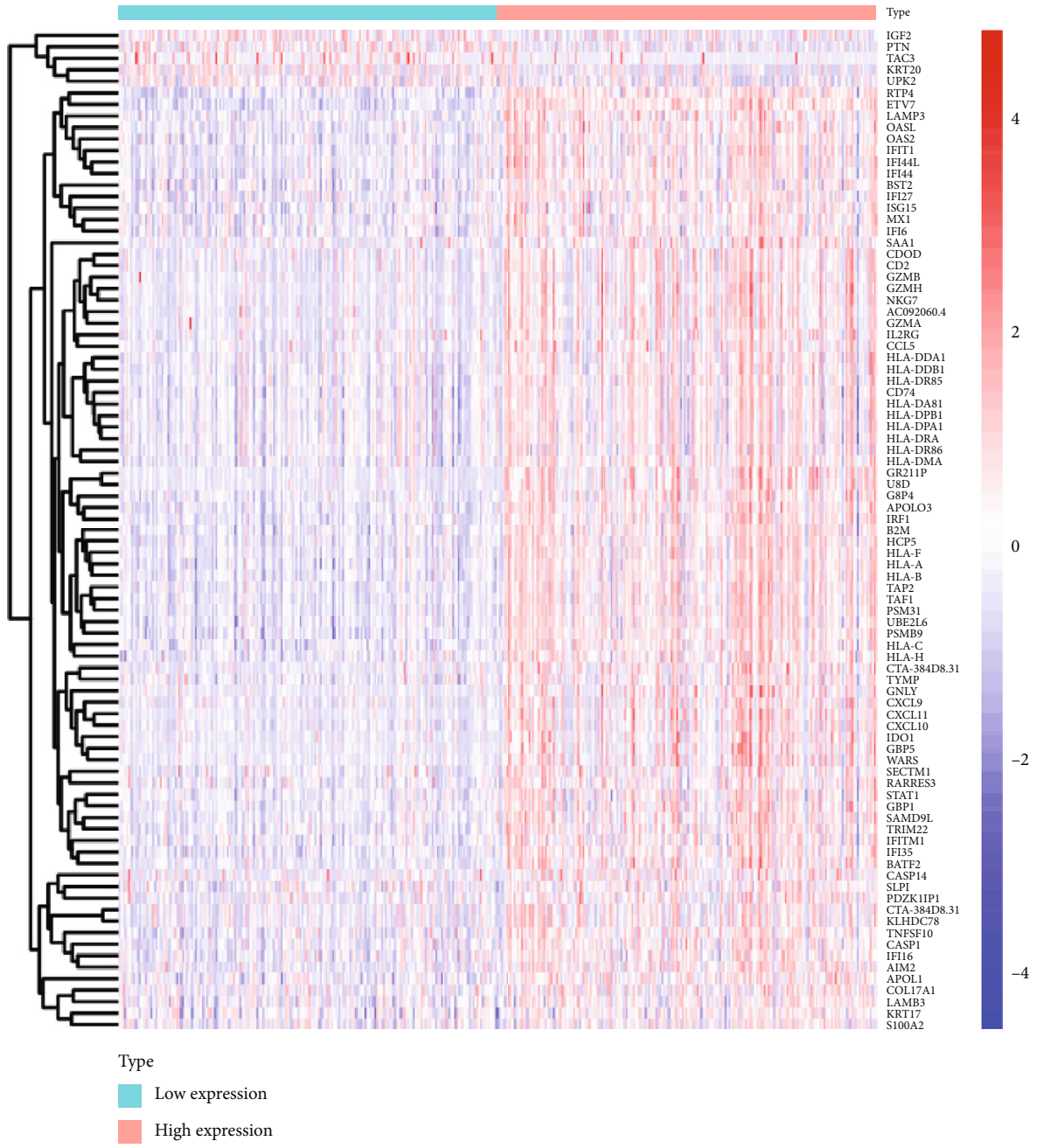

(b)

Figure 5: Continued. 


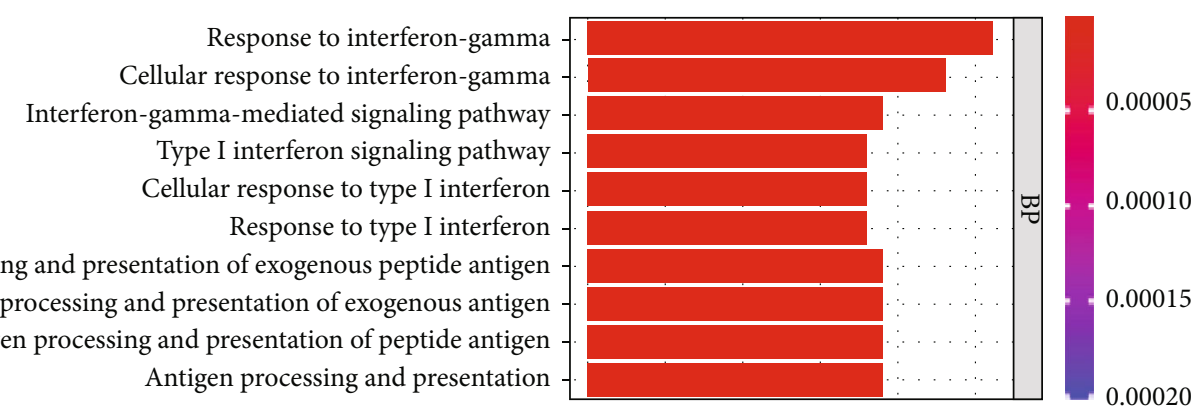

(c)

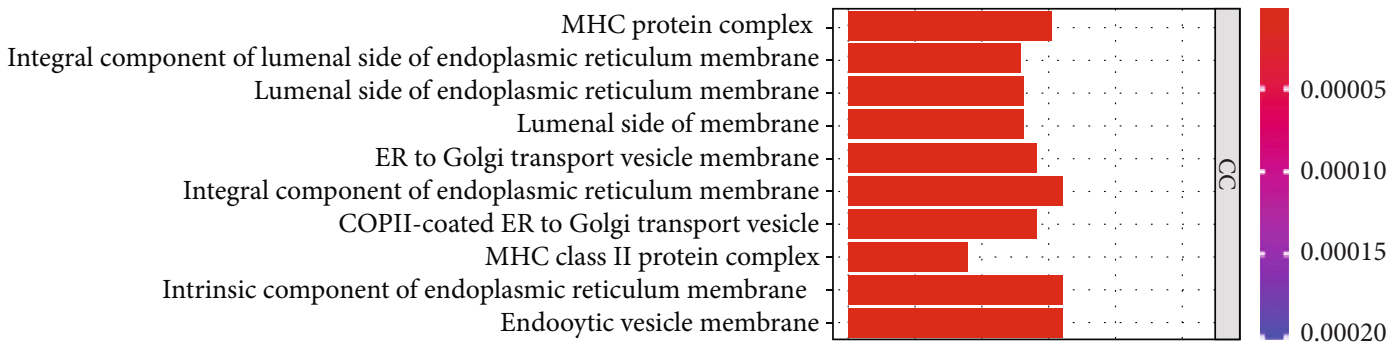

(d)

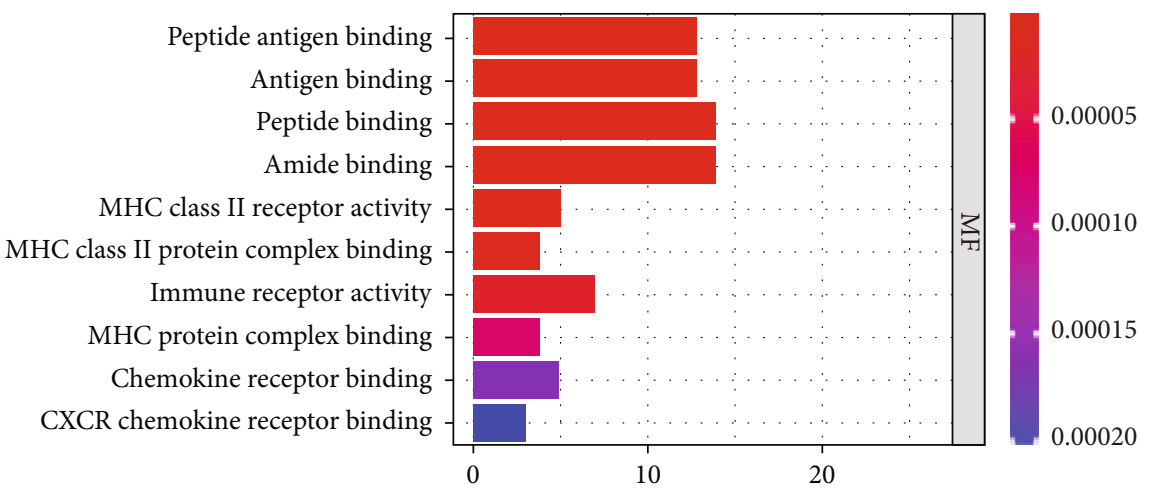

(e)

p.adjust

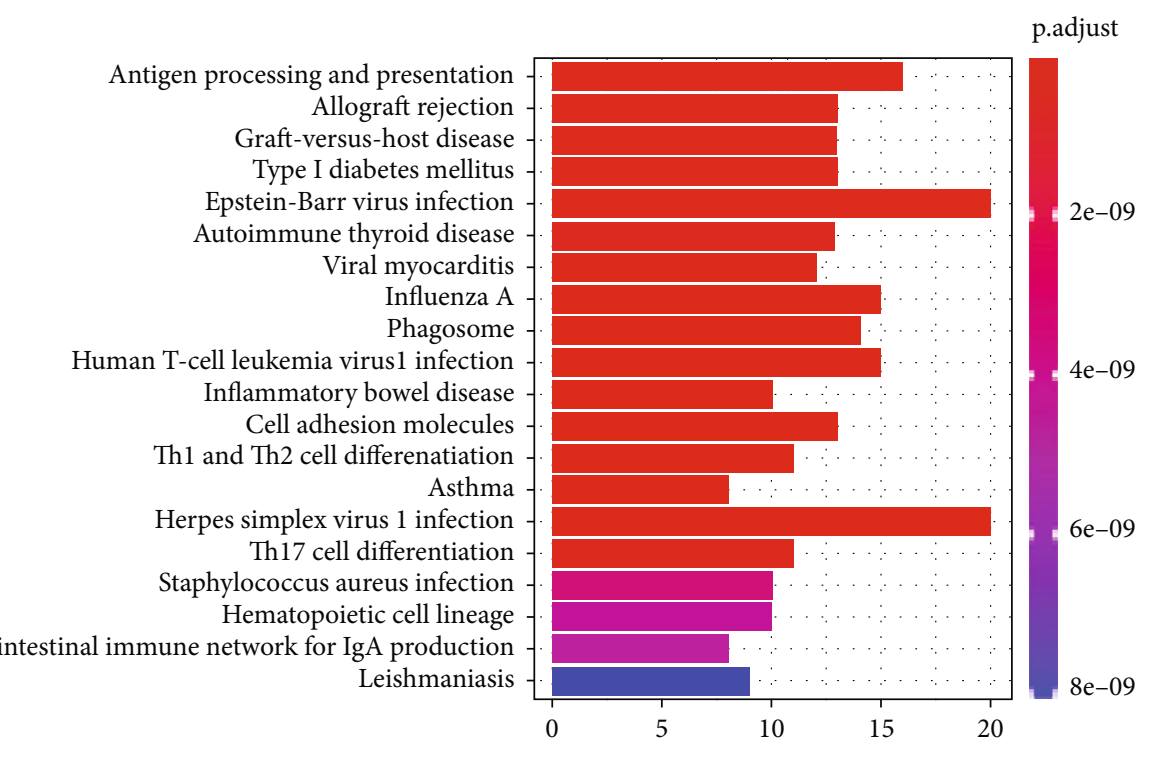

(f)

FIGURE 5: Continued. 

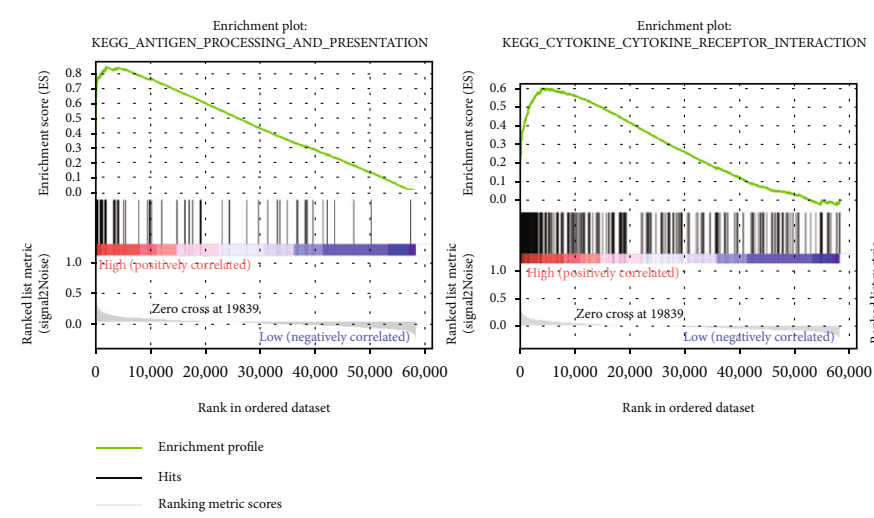

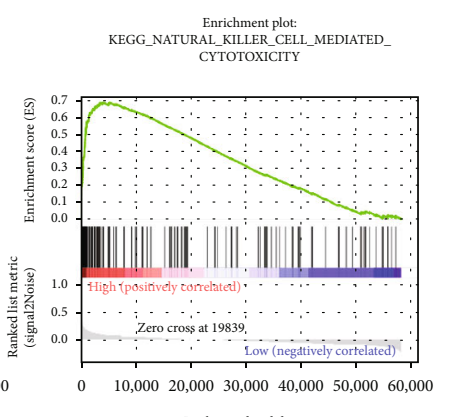

Rank in ordered dataset

$(\mathrm{g})$

FIgURe 5: Functionnal pathway enrichment of ETV7. (a). distribution of the DEGs in the low and high ETV7 groups. (b). heat map shows the expression levels of 87 DEGs in the high and low ETV7 groups. (c-e). GO analysis of ETV7 related genes 5F KEGG analysis of ETV7 related genes. (g). GSEA plots of "antigen processing and presentation," "natural killer cell mediated cytotoxicity," "JAK-STAT signaling pathway," and "cytokine-cytokine receptor interaction".

pathway" (Figure 5(c)). Primary terms within CCs included "MHC protein complex," "integral component of lumenal side of endoplasmic reticulum membrane," and "lumenal side of endoplasmic reticulum membrane" (Figure 5(d)). MFs ascribed to these DEGs included mainly "peptide antigen binding," "antigen binding," and several subterms of MHC (Figure 5(e)). Additionally, on KEGG analysis the DEGs were mainly enriched in "Antigen processing and presentation," "Allograft rejection," "Graft-versus-host disease," and "Type I diabetes mellitus" pathways (Figure 5(f)).

Surprisingly, in the GO-BP classification, immune response regulation and various immune cells (such as $\mathrm{T}$ cells, granulocytes, white blood cells, monocytes, and lymphocytes) were significantly enriched (Supplementary Table S9). Meanwhile, KEGG pathway analysis also uncovered that "Th1 and Th2 cell differentiation," "Th17 cell differentiation," and "natural killer cell mediated cytotoxicity" pathways were closely related to DEGs (Supplementary Table S10). Next, with the GSEA analysis, we were able to profile the pathways of the high-ETV7 expression samples. The results showed that high-ETV7 expression samples were mainly enriched in "antigen processing and presentation," "natural killer cell mediated cytotoxicity," "JAK-STAT signaling pathway," and "cytokine-cytokine receptor interaction" pathways (Figure 5(g)). Moreover, GSEA-GO analysis also showed that a large number of immune-related terms were substantially enriched. Detailed results of GSEA were shown in Supplementary Tables S11 and S12.

3.6. Analysis of Immune Landscape of ETV7-High and -Low Expression Group. The enrichment analysis showed the DEGs participated in a variety of immune-related terms and pathways; so, we analyzed the tumor microenvironment of BLCA.

ESTIMATE algorithm-derived immune scores ranged from -1658.36 to 3222.84 and stromal scores ranged from -2613.72 to 2173.23 (Supplementary Table S13). The average immune score of the high ETV7 subtype was higher than that of the low ETV7 subtype $(P=5 e-10)$, as well as the ESTIMATE score $(P=0.0043$, Figures 6(a)$6(c))$, which indicated that both scores were meaningfully correlated with ETV7.

The CIBERSORT algorithm demonstrated that tumors with high ETV7 were significantly associated with high fractions of T cells CD8, T cells CD4 memory activated, T cells follicular helper, and macrophages M1. In the low ETV7 group, there was a higher fraction of $\mathrm{B}$ cells naive, $\mathrm{B}$ cells memory, T cells CD4 naive, macrophages $\mathrm{M} 0$, and mast cells activated (Figures 6(d) and 6(e)).

\section{Discussion}

TFs are significant in modulating the progression and immune response of tumors. Unfortunately, the specific function of associated TFs in BLCA is rarely known. In this systematic analysis of TFs in BLCA, we first integrated the data from the TCGA and AnimalTFDB, and 13DE-TFs were found. In this current study, we correlate the 13DE-TFs to the immune infiltrating cells and strikingly found that ETV7 was unique and had the strongest positive correlation with T cells CD4 memory activated (Figure 2(c)). After profiles of ETV7 in BLCA, the result shown higher ETV7 expression in tumors than normal samples (Figures 3(a)3(c)). The correlation between ETV7 with clinicopathological parameters by UALCAN shown that the ETV7 was remarkably correlated with cancer stage. After preliminary study, this gene can be used as an independent prognostic factor. Indeed, all patients in various subgroups of BLCA with the high ETV7 expression showed a significantly shorter OS than those with the 1 ow ETV7 expression. Recent study reviewed that the ETV7 was a crucial prognostic factor in melanoma, which could potentially regulate the immune microenvironment [15], but its results from OS is opposite from our study. This may have to do with the specific function of genes in different tumor tissues.

ETV7 is a vital member of the ETS family, but its role in bladder cancer has been rarely reported. Our understanding 


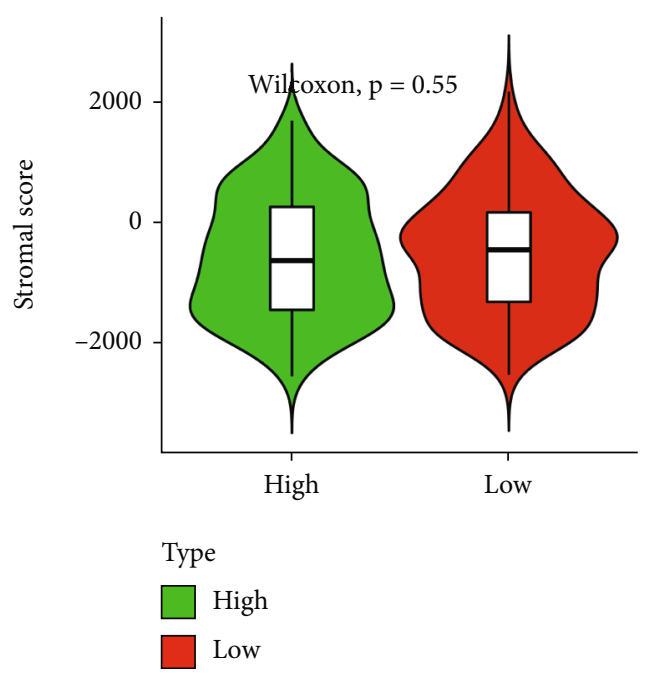

(a)

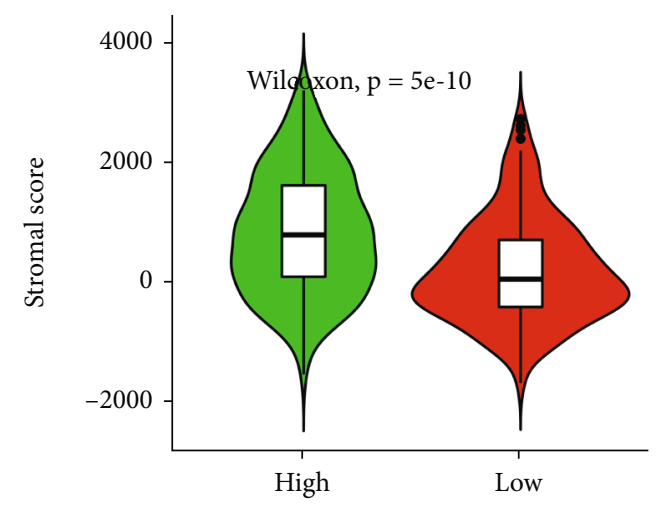

Type

$\square$ High

Low
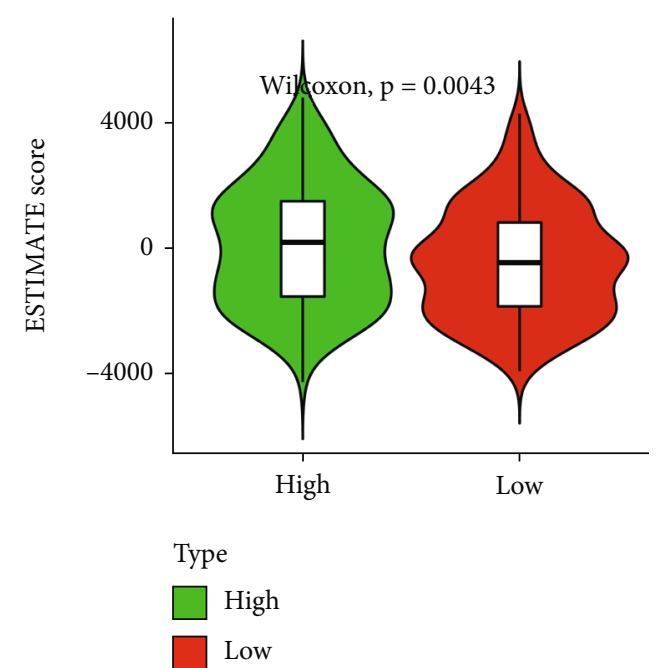

(c)

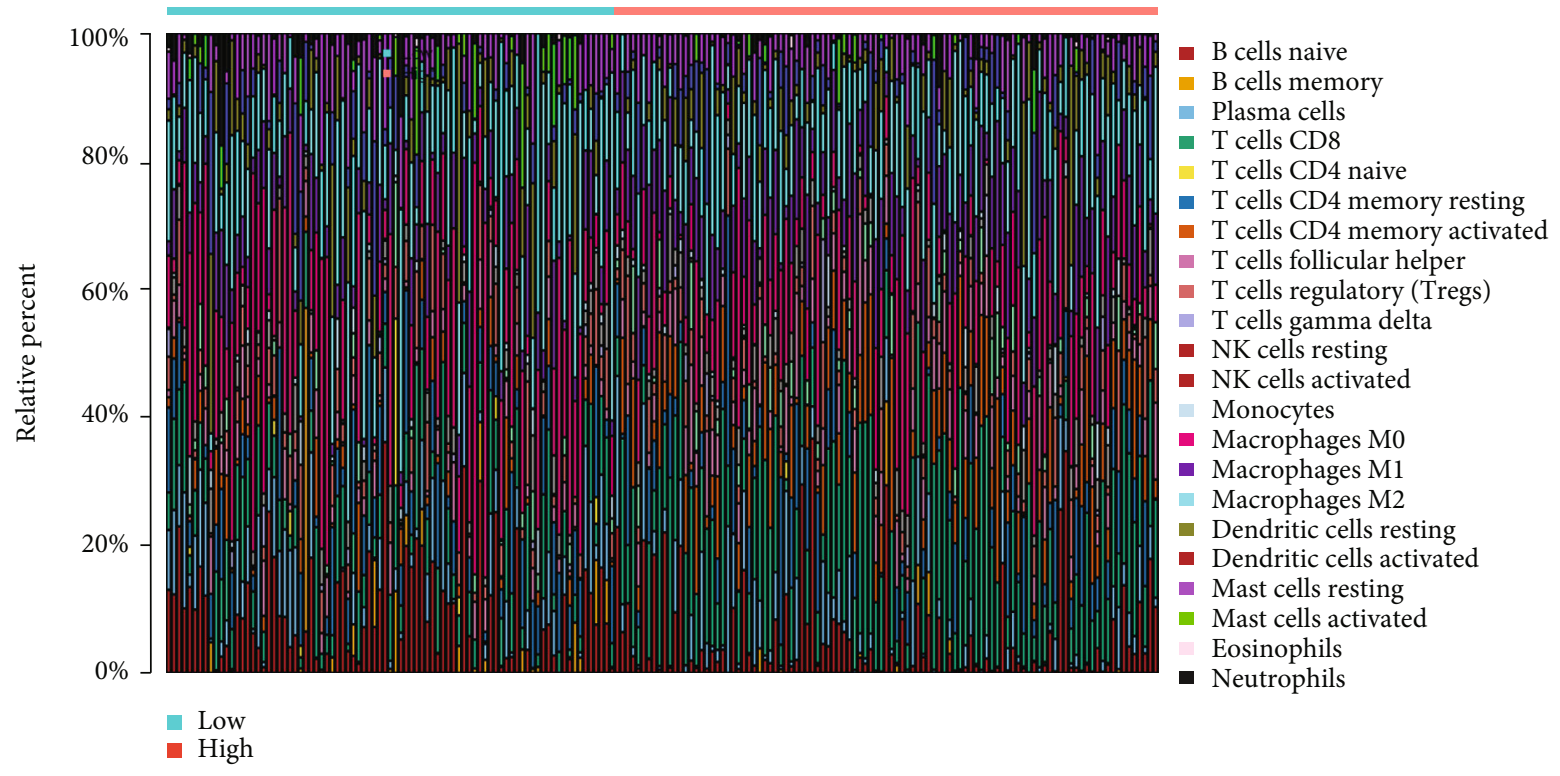

(d)

FIgUre 6: Continued. 


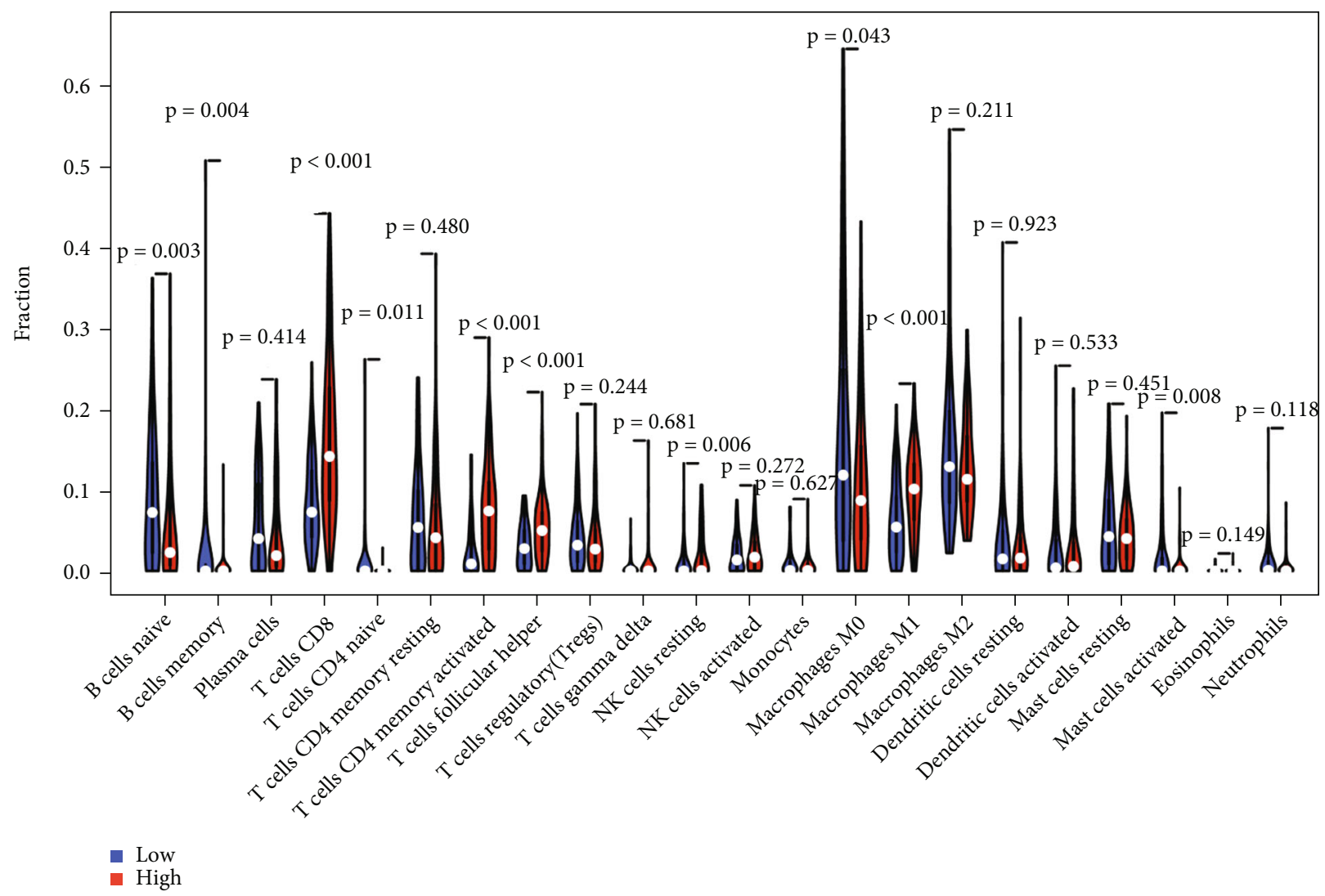

(e)

FIgURE 6: Immune landscape of ETV7 in various immune cells. (a)-(c) ESTIMATE algorithm immune, stromal, and estimate score. (d) Barplot showing the proportion of TICs in BLCA samples. Column names of plot were the sample's ID. (e) Violin plot showed the ratio differentiation of 21 kinds of immune cells between BLCA samples with low or high ETV7 expression relative to the median of ETV7 expression level, and Wilcoxon rank sum was used for the significance test.

of the role in pathology and physiology is rather limited. Previously, ETV7 was found to be highly associated with oncogene ETV6 [14]. Nevertheless, recent study shown the opposite function of two genes $[17,18]$. ETV has earlier been reported act as an essential component of a rapamycininsensitive mTOR complex in cancer [16].

To explore the role of ETV7 in BLCA, we divided the patients into low and high ETV7 groups and identified 87 DEGs (Figures 5(a) and 5(b), Supplementary Table S8). We analyzed 87 DGEs by conducting GO, KEGG, and GSEA analyses which are widely used bioinformatic tools in the functional location of specific genes. All of these enrichment analyses broad ETV7 involvement in immune related process and pathways, including the following: response to inteferon-gamma, cellular response to inteferon-gamma, inteferon-gamma-mediated signaling pathways, antigen processing and presentation, natural killer cell mediated cytotoxicity, JAK-STAT signaling pathway, MHC complex, and Th1, Th2, and Th17 cell differentiation. Th cell differentiation has been reported by nearly reports in melanoma which had a strong correlation with ETV7, and this is constant with our current study [15].The immune score of BLCA patients with high expression of ETV7 was significantly higher than that of
BLCA patients with low expression of ETV7, suggesting a higher degree of immune cell infiltration in the tumor microenvironment. Indeed, when the infiltration of various immune cells was estimated, the ETV7 showed strong positive correlation with high fraction of $\mathrm{T}$ cell $\mathrm{CD} 8$, $\mathrm{T}$ cell memory activated, $\mathrm{T}$ cell follicular helper and macrophages M1, which is similar to the previous study in melanoma [15].

In the low ETV7 group, there was a higher fraction of $\mathrm{B}$ cells naive, $\mathrm{B}$ cells memory $\mathrm{T}$ cells $\mathrm{CD} 4$ naive, macrophages M0, and Mast cell activated. These results confirm a strong association between ETV7 and T cell infiltration. In fact, in addition to find that ETV7 is closely related to the immune-related cell, process, and pathways, the KEGG and GO enrichment also showed the strong correlation with virus infection (HIV, HPV), and COVID-19 was also significantly enriched (Supplementary Table S9-S10); simultaneously, we found that ETV7 is involved in many intefron-related signaling pathway (Figure 5(c)). Indeed, recent study have found that ETV7 is an interferoninduced, repressive transcription factor that negatively regulates antiviral interferon-stimulated genes essential for controlling influenza virus and SARS-CoV-2 infections [32]. This result is in good agreement with our enrichment. Moreover, CSDC2 was identified a strong correlation with 
EMT pathway which can be associated with TIM in BLCA (Figure 2(b)), but it has not been investigated in BLCA microenvironment, we will continue to focus.

\section{Data Availability}

Expression data along with all available clinical information were retrieved for bladder cancer patients from The Cancer Genome Atlas (https://portal.gdc.cancer.gov/).Transcription factors were downloaded from AnimalTFDB database (http://bioinfo.life.hust.edu.cn/AnimalTFDB\#!/). Other data was also obtained from public database described in Materials and Methods.

\section{Conflicts of Interest}

The authors declare that there are no conflicts of interest.

\section{Authors' Contributions}

HM Li and SY Zheng conceived and designed. YB Zhang and $\mathrm{HM} \mathrm{Li}$ performed the experiments. $\mathrm{HM} \mathrm{Li}$ analyzed the data. L Wang, HM Li, and SY Zheng wrote the paper. All authors contributed to data analysis, drafting or revising the article, gave final approval of the version to be published, and agree to be accountable for all aspects of the work.

\section{Acknowledgments}

This work was financially supported by a grant from the National Natural Science Foundation of China (No. 81860494 to SY Zheng and 82060486 to R Zeng). We would like to thank for it.

\section{Supplementary Materials}

Supplementary 1. Table S1: 1665 human transcription factors from the AnimalTFDB. Table S2-S3: the upregulated and down-regulated DEGs of the TCGA-BLCA database. Table S4: 117 DE-TFs in bladder cancer. Table S5: 13 genes met the criteria for statistical significance via the $P<0.05$ among 117 DE-TFs in bladder cancer. Table S6-S7: univariate and multivariate analysis of ETV7. Table S8: a total of 87 DEGs (82 upregulated and 5 downregulated) were identified in the high ETV7 group. Table S9: enrichment of immune response regulation and various immune cells by GO-BP classification. Table S10: KEGG pathway analysis of DEGs. Table S11-12: detailed results of GSEA-GO analysis. Table S13: ESTIMATE algorithm-derived immune scores.

Supplementary 2. Figure S1: supplementary Figure 1: ETV7 expression in multiple database. (a) The differential expression of ETV7 between BLCA and normal tissues in GEO database. (b) ETV7 expression in MERAV database. (c) ETV7 expression levels in different cancer types in the TIMER database.

\section{References}

[1] R. Cao, B. Ma, L. Yuan, G. Wang, and Y. Tian, "Small nucleolar RNAs signature (SNORS) identified clinical outcome and prognosis of bladder cancer (BLCA)," Cancer Cell International, vol. 20, no. 1, p. 299, 2020.

[2] R. Y. Cao, L. Yuan, B. Ma, G. Wang, W. Qiu, and Y. Tian, “An EMT-related gene signature for the prognosis of human bladder cancer," Journal of Cellular and Molecular Medicine, vol. 24, no. 1, pp. 605-617, 2020.

[3] K. M. M. Johnson, N. R. Mahler, R. S. Saund et al., "role for the EWS domain of EWS/FLI in binding GGAA-microsatellites required for Ewing sarcoma anchorage independent growth," Proceedings of the National Academy of Sciences of the United States of America, vol. 114, no. 37, pp. 9870-9875, 2017.

[4] J. M. D.-V. Taylor, E. E. Dupont-Versteegden, J. D. Davies et al., "A role for the ETS domain transcription factor PEA3 in myogenic differentiation," Molecular and Cellular Biology, vol. 17, no. 9, pp. 5550-5558, 1997.

[5] C. H. Samakovlis, N. Hacohen, G. Manning, D. C. Sutherland, K. Guillemin, and M. A. Krasnow, "Development of the drosophila tracheal system occurs by a series of morphologically distinct but genetically coupled branching events," Development, vol. 122, no. 5, pp. 1395-1407, 1996.

[6] R. Treisman, "Ternary complex factors: growth factor regulated transcriptional activators," Current Opinion in Genetics \& Development, vol. 4, no. 1, pp. 96-101, 1994.

[7] N. K. Selvaraj, V. Kedage, and P. C. Hollenhorst, "Comparison of MAPK specificity across the ETS transcription factor family identifies a high-affinity ERK interaction required for ERG function in prostate cells," Cell Communication and Signaling, vol. 13, no. 1, 2015.

[8] C. D.-P. Charlot, H. Dubois-Pot, T. Serchov, Y. Tourrette, and B. Wasylyk, "A review of post-translational modifications and subcellular localization of Ets transcription factors: possible connection with cancer and involvement in the hypoxic response," Methods in Molecular Biology, vol. 647, pp. 3-30, 2010.

[9] I. Y. R. Luk, C. Reehorst, and J. Mariadason, "ELF3, ELF5, EHF and SPDEF transcription factors in tissue homeostasis and cancer," Molecules, vol. 23, no. 9, p. 2191, 2018.

[10] Y. W. Michael Hsing, P. S. Rennie, M. E. Cox, and A. Cherkasov, "ETS transcription factors as emerging drug targets in cancer," Medicinal Research Reviews, vol. 40, 2020.

[11] Q. Y. Cao, S. Yang, Q. Lv et al., "Five ETS family members, ELF-1, ETV-4, ETV-3L, ETS-1, and ETS-2 upregulate human leukocyte-associated immunoglobulin-like receptor-1 gene basic promoter activity," Aging, vol. 10, no. 6, pp. 1390-1401, 2018.

[12] M. G. Liu, W. Gao, J. C. van Velkinburgh, Y. Wu, B. Ni, and Y. Tian, "Role of Ets proteins in development, differentiation, and function of T-cell subsets," Medicinal Research Reviews, vol. 36, no. 2, pp. 193-220, 2016.

[13] H. J. Wang, S. Jain, P. Li et al., "Transcription factors IRF8 and PU.1 are required for follicular B cell development and BCL6driven germinal center responses," Proceedings of the National Academy of Sciences of the United States of America, vol. 116, no. 19, pp. 9511-9520, 2019.

[14] M. D. B. Potter, A. Buijs, B. Kreider, L. van Rompaey, and G. C. Grosveld, "Identification and characterization of a new human ETS-family transcription factor, TEL2, that is expressed in hematopoietic tissues and can associate with TEL1/ETV6," Blood, vol. 95, no. 11, pp. 3341-3348, 2000.

[15] H. Z. Qu, H. Zhao, X. Zhang et al., "Integrated analysis of the ETS family in melanoma reveals a regulatory role of ETV7 in 
the immune microenvironment," Frontiers in Immunology, vol. 11, p. 612784, 2020.

[16] F. C. K. G. Harwood, R. I. O'Hara, B. P. Cardone et al., "ETV7 is an essential component of a rapamycin-insensitive $\mathrm{mTOR}$ complex in cancer," Science Advances, vol. 4, no. 9, article eaar3938, 2018.

[17] P. R. Vivekanand and I. Rebay, "The SAM domain of human TEL2 can abrogate transcriptional output from TEL1 (ETV6) and ETS1/ETS2," PLoS One, vol. 7, no. 5, article e37151, 2012.

[18] H. M. C. Zhang, H. Chen, W. Liu et al., “AnimalTFDB: a comprehensive animal transcription factor database," Nucleic Acids Research, vol. 40, no. D1, pp. D144-D149, 2012.

[19] M. E. P. Ritchie, B. Phipson, D. Wu et al., "limma powers differential expression analyses for RNA-sequencing and microarray studies," Nucleic Acids Research, vol. 43, no. 7, article e47, 2015.

[20] C. J. H. Liu, F. F. Hu, M. X. Xia, L. Han, Q. Zhang, and A. Y. Guo, "GSCALite: a web server for gene set cancer analysis," Bioinformatics, vol. 34, no. 21, pp. 3771-3772, 2018.

[21] M. O. Uhlen, P. Oksvold, L. Fagerberg et al., "Towards a knowledge-based Human Protein Atlas," Nature Biotechnology, vol. 28, no. 12, pp. 1248-1250, 2010.

[22] D. S. B. Chandrashekar, B. Bashel, S. A. H. Balasubramanya et al., "UALCAN: a portal for facilitating tumor subgroup gene expression and survival analyses," Neoplasia, vol. 19, no. 8, pp. 649-658, 2017.

[23] K. Kumari, B. Das, A. K. Adhya, A. K. Rath, and S. K. Mishra, "Genome-wide expression analysis reveals six contravened targets of EZH2 associated with breast cancer patient survival," Scientific Reports, vol. 9, no. 1, p. 1974, 2019.

[24] Y. D. Shaul, B. Yuan, P. Thiru et al., "MERAV: a tool for comparing gene expression across human tissues and cell types," Nucleic Acids Research, vol. 44, no. D1, pp. D560-D566, 2016.

[25] T. Li, J. Fan, B. Wang et al., "TIMER: a web server for comprehensive analysis of tumor-infiltrating immune cells," Cancer Research, vol. 77, no. 21, pp. e108-e110, 2017.

[26] A. J. N. Gentles, A. M. Newman, C. L. Liu et al., "The prognostic landscape of genes and infiltrating immune cells across human cancers," Nature Medicine, vol. 21, no. 8, pp. 938945, 2015.

[27] A. M. S. Newman, C. B. Steen, C. L. Liu et al., "Determining cell type abundance and expression from bulk tissues with digital cytometry," Nature Biotechnology, vol. 37, no. 7, pp. 773782, 2019.

[28] A. M. Newman, C. L. Liu, M. R. Green et al., "Robust enumeration of cell subsets from tissue expression profiles," Nature Methods, vol. 12, no. 5, pp. 453-457, 2015.

[29] S. S. Jaleco, L. Swainson, V. Dardalhon, M. Burjanadze, S. Kinet, and N. Taylor, "Homeostasis of naive and memory $\mathrm{CD}^{+} \mathrm{T}$ cells: IL-2 and IL-7 differentially regulate the balance between proliferation and Fas-mediated apoptosis," Journal of Immunology, vol. 171, no. 1, pp. 61-68, 2003.

[30] M. O. R.-T. Kilinc, R. B. Rowswell-Turner, T. Gu, L. P. Virtuoso, and N. K. Egilmez, "Activated $\mathrm{CD}^{+}{ }^{+} \mathrm{T}$-effector/memory cells eliminate $\mathrm{CD} 4^{+} \mathrm{CD} 25^{+}$Foxp $3^{+}$T-suppressor cells from tumors via FasL mediated apoptosis," Journal of Immunology, vol. 183, no. 12, pp. 7656-7660, 2009.
[31] M. M. S. Opata and R. Stephens, "Chronic plasmodium chabaudi infection generates CD4 memory T cells with increased $\mathrm{T}$ cell receptor sensitivity but poor secondary expansion and increased apoptosis," Infect Immun, vol. 85, no. 3, 2017.

[32] H. M. Froggatt, A. T. Harding, R. R. Chaparian, and N. S. Heaton, "ETV7 limits antiviral gene expression and control of influenza viruses," Science Signaling, vol. 14, no. 691, 2021. 